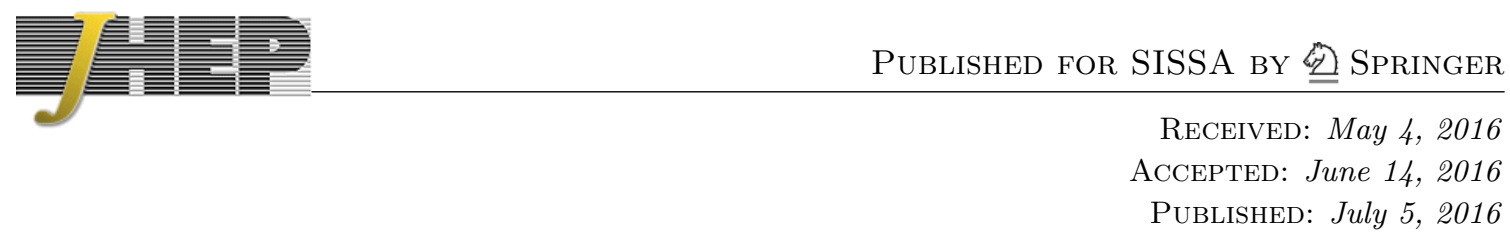

\title{
Towards a theory of nonassociative gravity
}

\author{
Ralph Blumenhagen and Michael Fuchs \\ Max-Planck-Institut für Physik (Werner-Heisenberg-Institut), \\ Föhringer Ring 6, 80805 München, Germany \\ E-mail: blumenha@mpp.mp.de, mfuchs@mpp.mpg.de
}

ABSTRACT: Violating the strong constraint of double field theory, non-geometric fluxes were argued to give rise to noncommutative/nonassociative structures. We derive in a rather pedestrian physicist way a differential geometry on the simplest nonassociative (phase-)space arising for a constant non-geometric $R$-flux. This provides a complementary presentation to the quasi-Hopf representation categorial one delivered by Barnes, Schenkel, Szabo in arXiv:1409.6331 + arXiv:1507.02792. As there, the notions of tensors, covariant derivative, torsion and curvature find a star-generalization. We continue the construction with the introduction of a star-metric and its star-inverse where, due to the nonassociativity, we encounter major deviations from the familiar structure. Comments on the Levi-Civita connection, a star-Einstein-Hilbert action and the relation to string theory are included, as well.

KEYwORDS: Non-Commutative Geometry, Flux compactifications

ArXiv EPrint: 1604.03253 


\section{Contents}

1 Introduction 2

2 Nonassociative star-product $\quad 4$

2.1 The universal $\mathcal{R}$-matrix and the associator $\phi \quad 4$

2.2 Derived tri-products 6

2.3 Scalars and the Leibniz rule $\quad 7$

2.4 Comment on star-scalars 8

3 The star-product on phase space $\quad 9$

3.1 Action on the basis 9

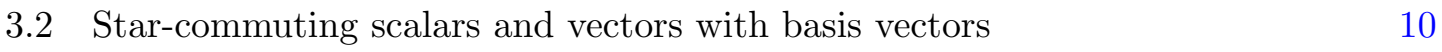

$\begin{array}{lll}3.3 & \text { Star-pairing between vectors and forms } & 12\end{array}$

4 The star-tensor calculus $\quad 13$

$\begin{array}{lll}4.1 & \text { Scalars } & 13\end{array}$

$\begin{array}{lll}4.2 & \text { Vectors and covectors } & 13\end{array}$

$\begin{array}{lll}4.3 & \text { Tensor product } & 15\end{array}$

4.4 Comment on $O(D, D)$ metric 16

$\begin{array}{lll}5 & \text { Nonassociative differential geometry } & 17\end{array}$

$\begin{array}{lll}5.1 \text { Covariant derivatives } & 17\end{array}$

$\begin{array}{ll}5.2 \text { Torsion } & 19\end{array}$

5.3 Riemann and Ricci tensor 20

6 Features of a star-metric $\quad 21$

6.1 Metric 22

6.2 The star-inverse 23

6.3 Comments on Levi-Civita connection 24

$\begin{array}{llr}7 & \text { Conclusions } & 27\end{array}$

$\begin{array}{lr}\text { A Hopf algebra approach } & 28\end{array}$

$\begin{array}{ll}\text { B Computing the Riemann tensor } & 29\end{array}$ 


\section{Introduction}

For the open string it is a long established result that its effective theory on a fluxed D-brane can be described by a noncommutative gauge theory. Whereas, for the closed string the appearance of a similar relation to noncommutative geometry is still under debate. In [1,2], indications were presented that support the picture that for nongeometric closed string backgrounds the coordinates do not commute, but give a noncommutative structure [2] for the case of so-called $Q$-flux and a nonassociative structure [1,2] for the case of non-geometric $R$-flux (see also the previous work [3] and the reviews [4, 5]). This nonassociativity for a constant $R$-flux background is captured by the commutation relations

$$
\left[x^{i}, x^{j}\right]=\frac{i l_{s}^{4}}{3 \hbar} R^{i j k} p_{k}, \quad\left[x^{i}, p_{j}\right]=i \hbar \delta^{i}{ }_{j}
$$

$p_{k}$ denotes the momentum and $i, j, k=1, \ldots, D$. More evidence for this result was delivered following various alternative approaches [6-11].

The string theoretic framework for nongeometric fluxes is double field theory (DFT) [12-15], an effective theory for the massless closed string modes that features manifest $O(D, D)$ symmetry and is a priori defined on a doubled space, where besides the usual coordinates $x^{i}$ one introduces so-called winding coordinates $\tilde{x}_{i}$. The latter can be considered as the canonical conjugate variables to the winding modes. For reviews of DFT please consult [16-18]. As one of its peculiar features, DFT is only consistent if one introduces a further constraint that reduces the degrees of freedom. For the fluctuations around a given background, this has to be the strong-constraint $\partial_{i} f \tilde{\partial}^{i} g+\tilde{\partial}^{i} f \partial_{i} g=0$ for every pair of fundamental objects $f$ and $g$. This implies that eventually the quantities depend on half of the coordinates.

It was pointed out in [19] that the nonassociative algebra above, presumes a violation of the strong constraint. In other words, one only has such a non-trivial structure, if the strong constraint between the background $R^{i j k}$ and fluctuations around it is violated. Since under these circumstances the background and the fluctuations are treated differently, this also presumes a background dependent version of DFT, similar to the one proposed in [20,21]. However, we emphasize that the question about the correct form of the DFT constraints is not completely settled yet. ${ }^{1}$

Once outside of Pandora's box, such a nonassociative structure has gained some interest also from the more formal noncommutative geometry point of view. In particular, in the framework of deformation quantization, a nonassociative star-product was introduced that realizes the above algebra [24-26]. Since it originates from the closed string, the expectation is that it might be possible to define a gravity theory on such a background. Based on Hopf algebra techniques, for the associative Moyal-Weyl star-product, a deformed formalism was developed [27-29] that allows to generalize all the concepts from differential geometry, like tensors, covariant derivative, torsion and curvature. The main idea is to introduce so-called star-diffeomorphisms and deform the Leibniz rule in such a way that the star-product of two star-tensors is again a star-tensor.

\footnotetext{
${ }^{1}$ An alternative proposal for a relation of DFT to a star-product in noncommutative geometry was presented in [22, 23].
} 
Based on earlier work [24-26], this latter framework was generalized recently by Barnes, Schenkel, Szabo [30-32] to so-called quasi-Hopf algebras, which are not any longer associative but whose associator is of a special form that, as an example, includes the constant $R$-flux star-product. The (to our taste) very formal, categorial approach followed in [30-32] led to the star-generalization of tensors, covariant derivative and curvature but stopped at the point where usually a metric and its Levi-Civita connection is introduced. Moreover, it is far from obvious whether all these structures have anything to do with string theory or DFT, respectively. If they do, then from string theory/DFT perspective the following issue needs to be resolved:

- At each order in $\alpha^{\prime}$ the string effective action is manifestly diffeomorphism invariant. (Similarly, the DFT action is invariant under generalized diffeomorphisms.) Therefore, one needs to understand how star-diffeomorphisms are related to these classical symmetries.

One possible way to resolve it may be the following observation made in [19] and further exploited in [33]. Since string theory is described on-shell by a two-dimensional conformal field, it was argued that on-shell any sign of nonassociativity should better be absent. That means that, using the equations of motion, the additional terms in the action resulting from the star-product should be total derivatives. Moreover, in [6] the CFT for a constant metric with a constant $R$-flux was constructed up to linear order in the $R$-flux. Via computing correlation functions of tachyon vertex operators a linear contribution was found that could be encoded in the nonassociative star-product from above. This means that comparing the two formalisms is expected to be reliable only up to linear order in the $R$-flux.

In this paper, even though we will not be able to fully clarify the above mentioned issue, as an intermediate step, we intend to provide a more pedestrian derivation of the structure of a nonassociative differential geometry. Avoiding abstract techniques from quasi-Hopf algebras, we investigate how a star-tensor calculus can be developed step by step. We will work on the entire phase space and will be able to construct a covariant derivative, the torsion and the curvature star-tensor. It is remarkable that this is still possible, even though, due to the nonassociativity, one has to be very careful with the bracketing in all of the expressions that appear in the course of the formal computations. However, when it comes to the introduction of a star-metric, its inverse and a Levi-Civita connection, we encounter a number of obstacles in the nonassociative case that were not present in the only noncommutative but associative case.

Concretely, this paper is organized as follows: in section 2 we introduce the nonassociative star-product for a non-vanishing $R$-flux and, after discussing some of its basis structures, we carry out the first steps towards a star-tensor formalism. Here we restrict to tensors only depending on configuration space. In section 3 we generalize some of these concepts to a full phase-space dependence, before in section 4 we develop the full tensor calculus. Section 5 is devoted to formulate the basic notions of a nonassociative differential geometry, i.e. we introduce a covariant derivative and define its torsion and curvature. In section 6 we move on and introduce a star-metric and discuss the appearing deviations from the usual structure once one wants to introduce a Levi-Civita connection and an Einstein-Hilbert action. 


\section{Nonassociative star-product}

In this section we provide some basic definitions and features of the nonassociative starproduct and introduce the concept of star-diffeomorphisms. The nonassociative starproduct is not completely generic in the sense that it does admit two important operators that control the way in which the product is noncommutative and nonassociative, respectively. In this first warm-up section, for simplicity we restrict ourselves to stardiffeomorphisms in configuration space $M$, i.e. those without an explicit momentum dependence. The generalization to the full phase space $\mathcal{M}=T^{*} M$ will be presented in section 3. As mentioned in the introduction, we will refrain from using the rather abstract Hopf algebra techniques from [24-26, 30-33], but try to build up the formalism step by step in an explicit way. To complete the picture, a brief introduction into quasi-Hopf algebras is presented in appendix A.

\subsection{The universal $\mathcal{R}$-matrix and the associator $\phi$}

In [24], a star-product was introduced that upon deformation quantization leads to the nonassociative algebra presented in the introduction. It can be considered as a nonassociative generalization of the Moyal-Weyl star-product. Thus, throughout this paper we will work with the star-product

$$
\begin{aligned}
f \star g & :=\cdot\left[\mathcal{F}^{-1}(f, g)\right] \\
& =\cdot\left[\exp \left(\frac{1}{2} i \hbar\left(\partial_{i} \otimes \tilde{\partial}_{p}^{i}-\tilde{\partial}_{p}^{i} \otimes \partial_{i}\right)+\frac{i l_{s}^{4}}{12 \hbar} R^{i j k}\left(p_{k} \partial_{i} \otimes \partial_{j}-\partial_{j} \otimes p_{k} \partial_{i}\right)\right) f \otimes g\right]
\end{aligned}
$$

which was suggested to capture the presence of a totally antisymmetric $R^{i j k}$-flux background in string theory. Note that in contrast to Moyal-Weyl, this star-product lives in the full phase space $\mathcal{M}$ containing derivatives in the space directions $\partial_{i}$ and the momentum directions $\tilde{\partial}_{p}^{i}$. We used the tensor product to indicate on which factor of $f \otimes g$ the derivatives act with the dot in front eventually turning the tensor products into usual multiplications. More explicitly, the star-product reads

$$
f \star g=f \cdot g+\frac{1}{2} i \hbar\left(\partial_{i} f \tilde{\partial}_{p}^{i} g-\tilde{\partial}_{p}^{i} f \partial_{i} g\right)+\frac{i l_{s}^{4}}{6 \hbar} R^{i j k} p_{k} \partial_{i} f \partial_{j} g+\ldots
$$

The first part of the product leads to the Heisenberg commutation relations when inserting $f=x^{i}$ and $g=p_{i}$ and the second part gives the non-trivial commutator between the coordinates

$$
\left[x^{i}, x^{j}\right]=\frac{i l_{s}^{4}}{3 \hbar} R^{i j k} p_{k}
$$

This product is nonassociative and violates the Jacobi identity. It was proposed in [26] that, when higher tensors are multiplied, the partial derivatives have to be replaced by Lie derivatives to guarantee compatibility with the exterior derivative. With $f, g$ now higher tensors, the star-product is defined as

$$
\begin{aligned}
f \star g=f \cdot g & +\frac{1}{2} i \hbar\left(\mathcal{L}_{\partial_{i}} f \mathcal{L}_{\tilde{\partial}_{p}^{i}} g+\mathcal{L}_{\tilde{\partial}_{p}^{i}} f \mathcal{L}_{\partial_{i}} g\right) \\
& +\frac{1}{2}\left(\frac{i l_{s}^{4}}{6 \hbar} R^{i j k} \mathcal{L}_{p_{k} \partial_{i}} f \mathcal{L}_{\partial_{j}} g-\frac{i l_{s}^{4}}{6 \hbar} R^{i j k} \mathcal{L}_{\partial_{j}} f \mathcal{L}_{p_{k} \partial_{i}} g\right) \ldots
\end{aligned}
$$

The non-trivial part is the $\mathcal{L}_{p_{k} \partial_{i}} f$ in the last line. 
The operator $\mathcal{F}^{-1}$ we defined in (2.1) is called the twist. As it is given by a phase its inverse $\mathcal{F}$ can be read off by switching the sign in the exponent. Due to the antisymmetry in the exponent a permutation of the arguments also inverts the twist

$$
\mathcal{F}^{-1}(f, g)=\mathcal{F}(g, f) .
$$

Using this we can deduce what happens to the star product under a permutation

$$
\begin{aligned}
f \star g & =\cdot\left[\mathcal{F}^{-1}(f, g)\right]=\cdot[\mathcal{F}(g, f)]=\cdot[\mathcal{F}^{-1} \underbrace{\mathcal{F} \mathcal{F}}_{:=\overline{\mathcal{R}}}(g, f)] \\
& :=\overline{\mathcal{R}}(g) \star \overline{\mathcal{R}}(f) .
\end{aligned}
$$

Here we introduced the universal $\mathcal{R}$-matrix $\mathcal{R}=\mathcal{F}^{-2}$ whose inverse $\overline{\mathcal{R}}=\mathcal{F}^{2}$ captures the extra factors appearing when one permutes scalars in the star-product. Therefore, it is a representation of the permutation group on this algebra. The notation in the second line of (2.6) means that first the $\overline{\mathcal{R}}$-matrix acts on $g$ and $f$ and afterwards the star-product is carried out. To see what is happening here, we evaluate (2.6) for functions $f, g \in C^{\infty}(M)$ on configuration space and up to linear order in the $R$-flux. For $f \star g$ we have

$$
f \star g=f g+\frac{i l_{s}^{4}}{6 \hbar} R^{i j k} p_{k} \partial_{i} f \partial_{j} g+\ldots
$$

and for $\overline{\mathcal{R}}(g) \star \overline{\mathcal{R}}(f)$

$$
\begin{aligned}
\overline{\mathcal{R}}(g) \star \overline{\mathcal{R}}(f) & =g \star f-2 \frac{i l_{s}^{4}}{6 \hbar} R^{i j k} p_{k} \partial_{i} g \star \partial_{j} f+\ldots \\
& =g f+\frac{i l_{s}^{4}}{6 \hbar} R^{i j k} p_{k} \partial_{i} g \partial_{j} f-2 \frac{i l_{s}^{4}}{6 \hbar} R^{i j k} p_{k} \partial_{i} g \partial_{j} f+\ldots \\
& =f g+\frac{i l_{s}^{4}}{6 \hbar} R^{i j k} p_{k} \partial_{i} f \partial_{j} g+\ldots
\end{aligned}
$$

The second important object is the associator $\phi$ which reorders the brackets in a product of three functions

$$
(f \star g) \star h=f^{\phi} \star\left(g^{\phi} \star h^{\phi}\right):=\left.f \star(g \star h)\right|_{\phi} .
$$

The inverse associator $\bar{\phi}$ similarly shifts brackets to the left. Again the associator is acting on the functions first and then the star product is executed. The associator is central and commutes with $\mathcal{F}$ and $\mathcal{R}$. Therefore expressions like $\mathcal{R}(f)^{\phi}=\mathcal{R}\left(f^{\phi}\right)$ are unambiguous. Explicit calculation gives the phase factor

$$
\phi(f, g, h)=\exp \left(\frac{l_{s}^{4}}{6} R^{i j k} \mathcal{L}_{\partial_{i}} \otimes \mathcal{L}_{\partial_{j}} \otimes \mathcal{L}_{\partial_{k}}\right)(f \otimes g \otimes h) .
$$

Thus (2.9) can be expressed in more detail as

$$
\begin{array}{r}
(f \star g) \star h=f \star(g \star h)+\sum_{n=1}^{\infty} \frac{1}{n !}\left(\frac{l_{s}^{4}}{6}\right)^{n} R^{i_{1} j_{1} k_{1}} \ldots R^{i_{n} j_{n} k_{n}}\left(\partial_{i_{1}} \ldots \partial_{i_{n}} f\right) \star \\
\left(\left(\partial_{j_{1}} \ldots \partial_{j_{n}} g\right) \star\left(\partial_{k_{1}} \ldots \partial_{k_{n}} h\right)\right) .
\end{array}
$$


For instance, for three functions $f, g, h \in C^{\infty}(M)$ on configuration space, we find the following terms up to second order in $R^{i j k}$

$$
\begin{aligned}
(f \star g) \star h= & f \star(g \star h) \\
& +\frac{l_{s}^{4}}{6} R^{i j k} \partial_{i} f \quad \partial_{j} g \quad \partial_{k} h \\
& +\frac{l_{s}^{4}}{6} \frac{i l_{s}^{4}}{6 \hbar} R^{i j k} R^{a b c} p_{c}\left(\partial_{i} f \partial_{a} \partial_{j} g \partial_{b} \partial_{k} h+\text { cycl. }\right) \\
& +\mathcal{O}\left(R^{3}\right) .
\end{aligned}
$$

The inverse associator $\bar{\phi}$ is obtained by switching the sign in the exponent. Due to the antisymmetry of $R^{i j k}$ also a permutation of the arguments inverts the twist. In this way the $\mathcal{R}$-matrix can invert the associator in our notation

$$
f^{\phi} \star\left(g^{\phi} \star h^{\phi}\right)=f^{\bar{\phi}} \star\left(\overline{\mathcal{R}}\left(h^{\bar{\phi}}\right) \star \overline{\mathcal{R}}\left(g^{\bar{\phi}}\right)\right)
$$

as we have $\phi(f, g, h)=\bar{\phi}(f, h, g)$.

Our nonassociative star-product is special in the sense that it admits the two operations $\mathcal{R}$ and $\phi$ that capture the effect of noncommutativity and nonassociativity. As we will see in section 2.3 , precisely these two extra operations allow one to still write down a generalized Leibniz rule for star-diffeomorphisms.

\subsection{Derived tri-products}

Clearly, in contrast to the star-product on the Moyal-Weyl plane, the most unconventional aspect of this star-product is that it involves the momentum coordinates. In [6], the CFT for a constant $R$-flux on a flat space allowed to extract information on a non-trivial triproduct on configuration space. The relation between the star- and the tri-product was suggested in [33].

Indeed, using the star-product (2.1), one can define so-called tri-products for functions on configuration space via

$$
\begin{aligned}
f_{1} \triangle f_{2} & \Delta \ldots \Delta f_{N}=\left.f_{1} \star\left(f_{2} \star\left(\ldots\left(f_{N-1} \star f_{N}\right) \ldots\right)\right)\right|_{p_{0}=0} \\
= & \cdot\left[\exp \left(-\frac{l_{s}^{4}}{12} \sum_{1 \leq a<b<c \leq N} R^{i j k} \partial_{i}^{a} \otimes \partial_{j}^{b} \otimes \partial_{k}^{c}\right)\left(f_{1} \otimes \ldots \otimes f_{N}\right)\right] .
\end{aligned}
$$

Note that after evaluating the nested star-product, one is restricting the result to the $p_{0}=0$ leaf. This implies e.g. $f_{1} \triangle f_{2}=f_{1} f_{2}$. These tri-products have the peculiar property that all $R$-flux dependent corrections become total derivatives so that the integral drastically simplifies as

$$
\int d^{d} x f_{1} \triangle f_{2} \triangle \ldots \triangle f_{N}=\int d^{d} x f_{1} f_{2} \ldots f_{N} .
$$

As proposed in [19], this fact can provide a way that nonassociativity of the underlying space-time can trivialize in the action. In [33] they also argue that string theory might be realized on the $p_{0}=0$ leaf, while deviations from $p_{0}=0$ correspond to membrane corrections. 


\subsection{Scalars and the Leibniz rule}

Following [27-29], in this section we take the first steps towards developing a star-tensor formalism. One defines a star-scalar as an object that transforms under a star-diffeomorphism, $\xi=\xi^{i}(x) \star \partial_{i}$ with the star-Lie derivative

$$
\delta_{\xi} f=\mathcal{L}_{\xi}^{\star} f:=\xi^{i} \star \partial_{i} f .
$$

We will consider only diffeomorphisms in the space directions first and discuss momentum diffeomorphisms with a full $\xi=\xi^{i} \star \partial_{i}+\tilde{\xi}_{i} \star \tilde{\partial}_{p}^{i}$ later. Next one demands that the star-product of two star-scalars should again be a star-scalar, i.e. we enforce

$$
\delta_{\xi}(f \star g)=\mathcal{L}_{\xi}^{\star}(f \star g)=\xi^{i} \star \partial_{i}(f \star g)=\xi^{i} \star\left(\partial_{i} f \star g\right)+\xi^{i} \star\left(f \star \partial_{i} g\right) .
$$

Here we used that the star product adds factors of the momentum but not of the coordinates such that the derivative can be pulled through. We can use the $\mathcal{R}$-matrix and the associator to bring this into the form of a generalized Leibniz rule. In the first term an inverse associator is enough while in the second term we find

$$
\begin{aligned}
\xi^{i} \star\left(f \star \partial_{i} g\right) & =\left.\left(\xi^{i} \star f\right) \star \partial_{i} g\right|_{\bar{\phi}} \\
& =\left.\left(\overline{\mathcal{R}}(f) \star \overline{\mathcal{R}}\left(\xi^{i}\right)\right) \star \partial_{i} g\right|_{\phi}=\left.\overline{\mathcal{R}}(f) \star\left(\overline{\mathcal{R}}\left(\xi^{i}\right) \star \partial_{i} g\right)\right|_{\phi^{2}} .
\end{aligned}
$$

From the first to the second line the permutation of the arguments inverts the associator similar to (2.13). From this we recognize

$$
\mathcal{L}_{\xi}^{\star}(f \star g)=\left(\mathcal{L}_{\xi^{\bar{\phi}}}^{\star} f^{\bar{\phi}}\right) \star g^{\bar{\phi}}+\overline{\mathcal{R}}\left(f^{\phi^{2}}\right) \star\left(\mathcal{L}_{\overline{\mathcal{R}}\left(\xi^{\phi^{2}}\right)} g^{\phi^{2}}\right) .
$$

Now we can proceed by defining higher star-tensors.

Vectors and covectors. Next, we require that $\omega_{i}=\partial_{i} f$ is a star-covector. The behavior under star-diffeomorphisms can be deduced as

$$
\delta_{\xi}\left(\partial_{i} f\right)=\partial_{i}\left(\delta_{\xi} f\right)=\xi^{j} \star\left(\partial_{j} \partial_{i} f\right)+\left(\partial_{i} \xi^{j}\right) \star\left(\partial_{j} f\right)
$$

so that a covector $\omega_{i}$ generically transforms as

$$
\delta_{\xi} \omega_{i}=\mathcal{L}_{\xi}^{\star} \omega_{i}=\xi^{j} \star\left(\partial_{j} \omega_{i}\right)+\left(\partial_{i} \xi^{j}\right) \star \omega_{j} .
$$

Following the general logic, a star-vector $v^{i}$ is defined via

$$
\delta_{\xi} v^{i}=\mathcal{L}_{\xi}^{\star} v^{i}=\xi^{j} \star\left(\partial_{j} v^{i}\right)-\overline{\mathcal{R}}\left(v^{j}\right) \star \overline{\mathcal{R}}\left(\partial_{j} \xi^{i}\right) .
$$

This guarantees that $f=v^{i} \star \omega_{i}$ transforms as a star-scalar. This gives rise to the definition of the star-commutator of star-vectors

$$
[v, w]_{\star}=v^{j} \star\left(\partial_{j} w^{i}\right)-\overline{\mathcal{R}}\left(w^{j}\right) \star \overline{\mathcal{R}}\left(\partial_{j} v^{i}\right)
$$

which is equal to the Lie-derivative. Clearly the commutator is manifestly $\overline{\mathcal{R}}$-antisymmetric $[v, w]_{\star}=-[\overline{\mathcal{R}}(v), \overline{\mathcal{R}}(w)]_{\star}$. This commutator could also be defined by $[,]_{\star}:=[,] \circ \mathcal{F}^{-1}$ making its covariance obvious through the similarity to the definition of the star-product. 
Tensors. Next we define the star-tensor-product of e.g. two star-vectors as $z=u \otimes_{\star} v$ with $z^{i j}=v^{i} \star w^{j}$. Extending the Leibniz rule (2.19) to the tensor product,

$$
\mathcal{L}_{\xi}^{\star}\left(v \otimes_{\star} w\right)=\left(\mathcal{L}_{\xi^{\phi}}^{\star} v^{\bar{\phi}}\right) \otimes_{\star} w^{\bar{\phi}}+\overline{\mathcal{R}}\left(u^{\phi^{2}}\right) \otimes_{\star}\left(\mathcal{L}_{\overline{\mathcal{R}}\left(\xi^{\phi^{2}}\right)}^{\star} w^{\phi^{2}}\right)
$$

one finds

$$
\mathcal{L}_{\xi}^{\star} z^{i j}=\xi^{k} \star\left(\partial_{k} z^{i j}\right)-\left(\partial_{k} \xi^{i}\right) \star z^{k j}-\left(\partial_{k} \xi^{j}\right) \star z^{i k} .
$$

The generalization to higher tensors is straightforward.

Composition and closure. When two Lie-derivatives act on an object we face a bracketing ambiguity. The correct solution lies in the commutator of the Lie-derivative. The closure property

$$
\left[\mathcal{L}_{\xi}^{\star}, \mathcal{L}_{\eta}^{\star}\right]_{\star} v=\mathcal{L}_{[\xi, \eta]_{\star}}^{\star} v
$$

is fulfilled if we define the commutator of two star-Lie-derivatives as

$$
\left[\mathcal{L}_{\xi}^{\star}, \mathcal{L}_{\eta}^{\star}\right]_{\star} v:=\mathcal{L}_{\xi^{\phi}}^{\star}\left(\mathcal{L}_{\eta^{\phi}}^{\star} v^{\phi}\right)-\mathcal{L}_{\overline{\mathcal{R}}\left(\eta^{\phi}\right)}^{\star}\left(\mathcal{L}_{\overline{\mathcal{R}}\left(\xi^{\phi}\right)}^{\star} v^{\phi}\right)
$$

This matches a known Hopf algebra result. There the consistent composition • of operators has the crucial property

$$
\left(O \bullet O^{\prime}\right)(z)=O^{\phi}\left(O^{\prime \phi}\left(z^{\phi}\right)\right) .
$$

This rule is very intuitive as the associator reorders the brackets in the usual way. This composition operator obviously enters the commutator of the Lie-derivatives and as we will see later also in the Riemann tensor. Thus the commutator is in general given by

$$
[A, B]=A \bullet B-\overline{\mathcal{R}}(B) \bullet \overline{\mathcal{R}}(A) .
$$

\subsection{Comment on star-scalars}

Since we have defined the Leibniz rules such that the star-product of two star-scalars is again a star-scalar, the question occurs whether an ordinary function $f(x) \in C^{\infty}(M)$ on configuration space is actually a star-scalar. To approach this question, let us consider the Taylor expansion of such a function

$$
f(x)=\sum_{n_{1}, \ldots, n_{k}=0}^{\infty} a_{n_{1}, \ldots, n_{k}} x_{1}^{n_{1}} \cdot \ldots \cdot x_{k}^{n_{k}} .
$$

Taking into account that $x_{i} \star x_{i}=x_{i} \cdot x_{i}$, we can define a corresponding star-Taylor expansion as

$$
f_{\star}(x)=\sum_{n_{1}, \ldots, n_{k}=0}^{\infty} a_{n_{1}, \ldots, n_{k}} x_{1}^{n_{1}} \star\left(x_{2}^{n_{2}} \star\left(\ldots \star x_{k}^{n_{k}}\right) \ldots\right) .
$$

Since the elementary, linear functions $h(x)=x_{i}$ are star-scalars by construction $f_{\star}(x)$ in (2.31) is a star-scalar, as well. Now we can define a subset of all star-scalars as $\mathfrak{F}=$ $\left\{f: f_{*}=f\right\}$, i.e. the set of those star-scalars, where the star-multiplications in the Taylor expansion act trivially. 
Let us show by induction that $\exp \left(q_{i} x^{i}\right) \in \mathfrak{F}$ with $q_{i}=$ const. Clearly, the linear function $g(x)=\vec{q} \cdot \vec{x} \in \mathfrak{F}$. Let us assume that $h_{n}(x)=(\vec{q} \cdot \vec{x})^{n} \in \mathfrak{F}$. We need to show that $g(x) \star h_{n}(x)=g(x) h_{n}(x)$. Since $g(x)$ is linear, the star-product simplifies to

$$
\begin{aligned}
g(x) \star h_{n}(x) & =g(x) h_{n}(x)+R^{i j k} \partial_{i} g(x) \partial_{j} h_{n}(x) p_{k} \\
& =g(x) h_{n}(x)+\left(R^{i j k} q_{i} q_{j} p_{k}\right) g(x) n h_{n-1}(x) \\
& =g(x) h_{n}(x) .
\end{aligned}
$$

Therefore, the tachyon vertex operators $\exp (\vec{q} \vec{x})$, from which features of the star-product were derived in [6], are indeed star-scalars. This can be considered as a self-consistency check. Now it is clear that every "sum" of such terms

$$
f(x)=\int d^{k} q \hat{f}(q) e^{q_{i} x^{i}}
$$

is also a star-scalar. Therefore, every $f(x) \in C^{\infty}(M)$ is a star-scalar as $f(x) \in \mathfrak{F}$.

\section{The star-product on phase space}

Since in the star-product (2.1) both the space coordinates and the momenta appear, it actually is defined on the phase space $\mathcal{M}$. Therefore the restriction to configuration space from the previous section is not very natural. Moreover, also vectors should have components along the full tangent space, including the momentum directions. As a splitting into space and momentum components results in a lot of terms, we introduce a doubled notation where $X_{I}=\left(\frac{p_{i}}{i \hbar}, x^{i}\right)$ and

$$
V=V^{I}(X) \star \partial_{I}=V^{i}(x, p) \star \partial_{i}+\tilde{V}_{i}(x, p) \star i \hbar \tilde{\partial}_{p}^{i} .
$$

Similar to double field theory, a sum over a capital index $I=1, \ldots, 2 D$ always runs over an upper and a lower index. For revealing its full contents we will present most formulas in both a doubled and in a split-into-components notation. In this section we will derive several useful formulas that will be used in the next section for the construction of a nonassociative differential geometry calculus.

\subsection{Action on the basis}

Recall that in the star-product (2.4) several Lie-derivaties appear which usually reduce to partial derivaties for functions. More concretely we have

$$
\begin{aligned}
\mathcal{L}_{\partial_{i}} & :=P_{i}, \\
i \hbar \mathcal{L}_{\tilde{\partial}_{p}^{i}} & :=\tilde{P}^{i}, \\
\frac{i l_{s}^{4}}{6 \hbar} R^{i j k} \mathcal{L}_{p_{j} \partial_{k}} & :=M^{i} .
\end{aligned}
$$

$P$ and $\tilde{P}$ denote translations in space and momentum directions, while $M$ induces socalled Bobb-shifts in the momentum directions. Using these operators we can rewrite the 
star-product as

$$
f \star g=\cdot\left[\exp \left(\frac{1}{2}\left(P_{\mu} \otimes \tilde{P}^{\mu}-\tilde{P}^{\mu} \otimes P_{\mu}\right)+\frac{1}{2}\left(M^{\mu} \otimes P_{\mu}-P_{\mu} \otimes M^{\mu}\right)\right) f \otimes g\right] .
$$

In doubled notation we can merge the operators from (3.2) into

$$
\begin{aligned}
P^{I} & =\left(\tilde{P}^{i}, P_{i}\right)=\left(i \hbar \tilde{\mathcal{L}}_{\partial_{p}^{i}}, \mathcal{L}_{\partial_{i}}\right), \\
M^{I} & =-F^{I J K} X_{J} P_{K}=\left(\frac{i l_{s}^{4}}{6 \hbar} \mathcal{L}_{R^{i j k} p_{j} \partial_{k}}, 0\right),
\end{aligned}
$$

where $F^{I J K}$ has only one non-vanishing component $F^{i j k}=\frac{l_{s}^{4}}{6} R^{i j k}$. The fact that these operators contain Lie-derivatives implies a non-trivial action on the fundamental forms $d x^{i}$, $d p_{i}$. Merging them into $d X^{I}=\left(d x^{i}, \frac{d p_{i}}{i \hbar}\right)$ and using $[\mathcal{L}, d]=0$, one obtains

$$
\begin{aligned}
P^{I}\left(d X^{J}\right) & =0, \\
M^{I}\left(d X^{J}\right) & =F^{I J K} d X_{K} \quad \text { or } \quad M^{i}\left(d x^{j}\right)=\frac{l_{s}^{4}}{6} R^{i j k} \frac{d p_{k}}{i \hbar} .
\end{aligned}
$$

By duality between tangent and cotangent space or by acting on $\delta_{J}^{I}=\partial_{J} \star d x^{I}=\partial_{j} \star$ $d x^{i}+i \hbar \partial_{p}^{j} \star \frac{d p_{i}}{i \hbar}$ one has the relations

$$
\begin{aligned}
P^{I}\left(\partial^{J}\right) & =0, \\
M^{I}\left(\partial^{J}\right) & =F^{I J K} \partial_{K} \quad \text { or } \quad M^{i}\left(i \hbar \tilde{\partial}_{p}^{j}\right)=\frac{l_{s}^{4}}{6} R^{i j k} \partial_{k} .
\end{aligned}
$$

Note that $e^{I}=\partial^{I}$ is considered here as a basis of the tangent space. Let us make the following three observation:

- Since the $P_{i}$ act trivially onto any basis vector, the associator becomes the identity when acting on a basis vector. We will refrain from writing brackets in an expression like $A \star B \star \partial_{I}$ when both bracketings are equal.

- However, the $M^{i}$ and therefore the $\star$-product itself and the $\mathcal{R}$-matrix act non-trivially on the basis vector $\tilde{\partial}_{p}$.

- Due to $M\left(M\left(\tilde{\partial}_{p}\right)\right)=M(\partial)=0$ the star-product terminates after the first order when acting on any basis vector.

The main task in the following is to take this non-trivial action of $M^{i}$ into account when developing the star-tensor calculus and a star-differential geometry. Since there are only first order corrections, this is still feasible.

\subsection{Star-commuting scalars and vectors with basis vectors}

Let us now consider expressions like $f(X) \star \partial_{I}$. For any arbitrary function $f=f(x, p)$ we trivially find ${ }^{2}$

$$
f \star \partial_{i}=f \cdot \partial_{i} \quad \Rightarrow \quad f \star \partial_{i}-\partial_{i} \star f=0,
$$

\footnotetext{
${ }^{2}$ Note that, in contrast to $\partial_{i} f$, the derivative in $\partial_{i} \star f=e_{i} \star f$ is not meant to act on $f$.
} 
but an additional term arises when expanding the star-product (3.3) in

$$
f \star i \hbar \tilde{\partial}_{p}^{i}=f \cdot i \hbar \tilde{\partial}_{p}^{i}-\frac{1}{2} P_{j} f \cdot M^{j}\left(i \hbar \tilde{\partial}_{p}^{i}\right)=f \cdot i \hbar \tilde{\partial}_{p}^{i}+\frac{l_{s}^{4}}{12} R^{i j k} \partial_{j} f \star \partial_{k}
$$

so that

$$
f \star i \hbar \tilde{\partial}_{p}^{i}-i \hbar \tilde{\partial}_{p}^{i} \star f=\frac{l_{s}^{4}}{6} R^{i j k} \partial_{j} f \star \partial_{k} .
$$

In doubled notation this can be written as

$$
f \star \partial^{I}-\partial^{I} \star f=F^{I J K} \partial_{J} f \star \partial_{K}
$$

By comparing (3.5) with (3.6) we see that the star-product acts in the same way on $d x^{I}$ and $\partial^{I}$. The relation (3.10) must therefore also hold for the basis forms

$$
f \star d x^{I}-d x^{I} \star f=F^{I J K} \partial_{J} f \star d x_{K} .
$$

From (3.10) we see that the two possibilities to form a vector, $V^{I} \star \partial_{I}$ and $\partial_{I} \star V^{I}$, are not equivalent. As will be explained later we use the first convention. This has also consequences for the right and left multiplication of a scalar and a vector. While for the left multiplication one gets

$$
f \star\left(V^{I} \star \partial_{I}\right)=\left(f \star V^{I}\right) \star \partial_{I},
$$

since the associator vanishes on basis vectors but the right multiplication gives an additional term

$$
\left(V^{I} \star \partial_{I}\right) \star f=\left(V^{I} \star f\right) \star \partial_{I}-F^{I J K}\left(V_{I} \star \partial_{J} f\right) \star \partial_{K} .
$$

The non-trivial action onto the basis vector also has consequences for the derivation of the Leibniz rule. Recall that in (2.17) we were exchanging the order of a basis vector $e_{i}=\partial_{i}$ and a scalar. Therefore, using (3.10) we can derive the rule

$$
\partial^{I}(f \star g)=\partial^{I} f \star g+f \star \partial^{I} g-F^{I J K} \partial_{J} f \star \partial_{K} g .
$$

The same formulas continue to hold more generally for the operators $P^{I}$ where the partial derivatives are replaced by Lie-derivatives. The commutator with the Bopp-shifts is simply

$$
\left[P^{I}, M^{J}\right]=F^{I J K} P_{K},
$$

reproducing the action on the basis vectors (3.6). With this commutator at hand one can calculate the Leibniz rule for $M^{I}$

$$
M^{I}(f \star g)=M^{I} f \star g+f \star M^{I} g+F^{I J K} \partial_{J} f \star \partial_{K} g .
$$

Finally, the non-trivial star-commutators between the basis vectors and the scalars or vectors have non-trivial consequences when an expression involves the action of the $\mathcal{R}$ matrix. Looking back one realizes that the structure of the additional terms arising from 
the action on the basis vectors can essentially be deduced immediately from the index structure. For the $\mathcal{R}$ matrix acting on a scalar and a vector, one can derive the relation

$$
\overline{\mathcal{R}}(f) \otimes \overline{\mathcal{R}}\left(V^{I} \star \partial_{I}\right)=\overline{\mathcal{R}}(f) \otimes \overline{\mathcal{R}}\left(V^{I}\right) \star \partial_{I}+F^{I J K} \overline{\mathcal{R}}\left(\partial_{I} f\right) \otimes \overline{\mathcal{R}}\left(V_{J}\right) \star \partial_{K},
$$

and by iteration we find for the interchange of two vectors

$$
\begin{aligned}
\overline{\mathcal{R}}\left(V^{I} \star \partial_{I}\right) \otimes \overline{\mathcal{R}}\left(U^{J} \star \partial_{J}\right)= & \overline{\mathcal{R}}\left(V^{I}\right) \star \partial_{I} \otimes \overline{\mathcal{R}}\left(U^{J}\right) \star \partial_{J} \\
& -F^{M N I} \overline{\mathcal{R}}\left(V_{N}\right) \star \partial_{I} \otimes \overline{\mathcal{R}}\left(\partial_{M} U^{J}\right) \star \partial_{J} \\
& +F^{M N J} \overline{\mathcal{R}}\left(\partial_{M} V^{I}\right) \star \partial_{I} \otimes \overline{\mathcal{R}}\left(U_{N}\right) \star \partial_{J} \\
& -F^{A B I} F^{M N J} \mathcal{R}\left(\partial_{M} V_{B}\right) \star \partial_{I} \otimes \mathcal{R}\left(\partial_{A} U_{N}\right) \star \partial_{J}
\end{aligned}
$$

As we will see in section 4, even though this non-trivial action onto the basis vectors will produce a lot of additional terms, they will nevertheless organize themselves perfectly well so that the form of the star-tensor relations from section 2 remain the same. ${ }^{3}$

\subsection{Star-pairing between vectors and forms}

Let us move on and define a star-pairing between star-vectors and star-forms. Eventually, this should result in a contraction of the components with a star-product in between, i.e.

$$
V^{I} \star \omega_{I}=V^{i} \star \omega_{i}+\tilde{V}_{i} \star \tilde{\omega}^{i}
$$

First, let us define the product between the basis vectors and forms in the easiest way.

$$
\partial_{I} \star d x^{J}=d x^{J} \star \partial_{I}=\delta_{I}^{J}
$$

In order to obtain the intuitive contraction (3.19) as a consequence of this basis vector multiplication, we need the following convention for forms and vectors. In star-vectors the basis vectors must be on the right and in forms they must be on the left

$$
V=V^{I} \star \partial_{I}, \quad \omega=d x^{I} \star \omega_{I} .
$$

Only then we indeed find

$$
V \star \omega=\left(V^{I} \star \partial_{I}\right) \star\left(d x^{J} \star \omega_{J}\right)=V^{I} \star \delta_{I}^{J} \star \omega_{J}=V^{I} \star \omega_{I} .
$$

This convention for forms and vectors corresponds to the mathematical definition of the contraction

$$
\begin{aligned}
\langle,\rangle_{\star}: T M \otimes_{\star} T^{*} M & \rightarrow \mathbb{R}, \\
V \otimes_{\star} \omega & \rightarrow\langle,\rangle \circ \mathcal{F}^{-1}(V, \omega)=V^{I} \star \omega_{I}
\end{aligned}
$$

where the first entry is reserved for the vector and the second one for the form.

\footnotetext{
${ }^{3}$ Such a nice behavior was shown in [30, 31] for arbitrary quasi-Hopf algebras.
} 


\section{The star-tensor calculus}

In this and the next section we will develop the basic notions of a nonassociative differential geometry on the full twisted phase space. From a more mathematical perspective this was already done in [31] for arbitrary quasi-Hopf algebras. The purpose here is to show rather explicitly how this concretely works for the star-product (2.1). We first repeat the procedure from section 2.3 and develop the notion of star-diffeomorphisms and its corresponding tensors. Here we need to rely on the relations derived in the previous section.

\subsection{Scalars}

We define a star-scalar $f=f(x, p)$ to be a quantity transforming under twisted diffeomorphisms generated by a vector $\xi=\xi^{I}(X) \star \partial_{I}=\xi^{i}(x, p) \star \partial_{i}+\tilde{\xi}_{i}(x, p) \star i \hbar \tilde{\partial}_{p}^{i}$ as

$$
\delta_{\xi} f=\mathcal{L}_{\xi}^{\star} f=\xi^{I} \star \partial_{I} f=\xi^{i} \star \partial_{i} f+\tilde{\xi}_{i} \star i \hbar \tilde{\partial}_{p}^{i} f .
$$

This definition is the reason for our choice of convention $V^{I} \star \partial_{I}$ instead of $\partial_{I} \star V^{I}$. We demand the star-product of two scalars to be a star-scalar again. Switching the partial derivatives through with (3.14) gives

$$
\begin{aligned}
\mathcal{L}_{\xi}^{\star}(f \star g) & =\xi^{I} \star \partial_{I}(f \star g) \\
& =\xi^{I} \star\left(\partial_{I} f \star g\right)+\xi^{I} \star\left(f \star \partial_{I} g\right)-F^{I J K} \xi_{I} \star\left(\partial_{J} f \star \partial_{K} g\right) .
\end{aligned}
$$

Utilizing the relation (3.17), this expression including the $F^{I J K}$-correction term can be compactly written as

$$
\mathcal{L}_{\xi}^{\star}(f \star g)=\left.\left(\mathcal{L}_{\xi}^{\star} f\right) \star g\right|_{\bar{\phi}}+\left.\overline{\mathcal{R}}(f) \star\left(\mathcal{L}_{\overline{\mathcal{R}}(\xi)}^{\star} g\right)\right|_{\phi^{2}} .
$$

The Lie-derivative $\mathcal{L}^{*}$ therefore still obeys the same Leibniz rule as in (2.19). The extra terms arising from the non-trivial commutators with the basis vectors conspire in such a way that the formal expression for the Leibniz rule does not change. This is quite a remarkable feature.

\subsection{Vectors and covectors}

Guided by (2.23) we define the transformation of a vector $V$ with the twisted commutator

$$
\delta_{\xi} V=\mathcal{L}_{\xi}^{\star} V=[\xi, V]_{\star}:=[,] \circ \mathcal{F}^{-1} .
$$

This definition guarantees a covariant behavior under star multiplications and reads

$$
\begin{aligned}
\mathcal{L}_{\xi}^{\star} V & =\xi(V)-\overline{\mathcal{R}}(V)(\overline{\mathcal{R}}(\xi)) \\
& =\xi^{I} \star \partial_{I} V^{J} \star \partial_{J}-\overline{\mathcal{R}}(V)^{I} \star \partial_{I}\left(\overline{\mathcal{R}}(\xi)^{J}\right) \star \partial_{J} .
\end{aligned}
$$

In (3.18) we computed how the $\mathcal{R}$-matrix acts on two vectors. Inserting this in the definition of the commutator (4.5) gives in components

$$
\begin{aligned}
\mathcal{L}_{\xi}^{\star} V^{I}= & \xi^{J} \star \partial_{J} V^{I}-\overline{\mathcal{R}}\left(V^{J}\right) \star \partial_{J}\left(\overline{\mathcal{R}}\left(\xi^{I}\right)\right) \\
& -\overline{\mathcal{R}}\left(\partial_{M} V^{J}\right) \star \partial_{J}\left(\overline{\mathcal{R}}\left(\xi_{N}\right)\right) F^{M N I} .
\end{aligned}
$$

Here, we did not pull the $\mathcal{R}$-matrices out of the momentum derivatives as more terms proportional to $F^{I J K}$ would arise. 
Next we verify the closure property of two star-Lie-derivatives acting on a star-scalar

$$
\left[\mathcal{L}_{\xi}^{\star}, \mathcal{L}_{\eta}^{\star}\right]_{\star} f=\left.\xi(\eta(f))\right|_{\phi}-\left.\overline{\mathcal{R}}(\eta)(\overline{\mathcal{R}}(\xi)(f))\right|_{\phi}=\mathcal{L}_{[\xi, \eta]_{\star}}^{\star} .
$$

For this purpose we compute

$$
\begin{aligned}
(\xi \bullet \eta) f= & \xi^{\phi}\left(\eta^{\phi} f^{\phi}\right)=\left.\xi^{I} \star \partial_{I}\left(\eta^{J} \star \partial_{J} f\right)\right|_{\phi} \\
= & \left(\xi^{I} \star \eta^{J}\right) \star \partial_{I} \partial_{J} f+\left(\xi^{I} \star \partial_{I} \eta^{J}\right) \star \partial_{J} f \\
& -F^{I J K}\left(\xi_{I} \star \partial_{J} \eta^{A}\right) \star \partial_{K} \partial_{A} f
\end{aligned}
$$

and

$$
\begin{aligned}
(\overline{\mathcal{R}} \eta \bullet \overline{\mathcal{R}} \xi) f= & \left(\xi^{J} \star \eta^{I}\right) \star \partial_{J} \partial_{I} f+\left(\overline{\mathcal{R}}\left(\eta^{I}\right) \star \partial_{I} \overline{\mathcal{R}}\left(\xi^{J}\right)\right) \star \partial_{J} f \\
& +\left(F^{M N J} \overline{\mathcal{R}}\left(\partial_{M} \eta^{I}\right) \star \partial_{I} \overline{\mathcal{R}}\left(\xi_{N}\right)\right) \star \partial_{J} f \\
& +\left(F^{M N J} \xi_{N} \star\left(\partial_{M} \eta^{I}\right)\right) \star \partial_{I} \partial_{J} f
\end{aligned}
$$

Adding both terms, remarkable cancellations occur that finally yield (4.7). Although intuitive it is tedious to verify the Leibniz rule

$$
\left[U,[V, W]_{\star}\right]_{\star}=\left.\left[[U, V]_{\star}, W\right]_{\star}\right|_{\bar{\phi}}+\left.\left[\overline{\mathcal{R}}(V),[\overline{\mathcal{R}}(U), W]_{\star}\right]_{\star}\right|_{\phi^{2}}
$$

that is nothing else than the star-Jacobi identify for three star-vectors. This relation can be found more easily with Hopf algebra techniques as in [33].

Next we come to the definition of a star-covector $\omega=d x^{I} \star \omega_{I}$. Like in section 2 , its star-Lie-derivative can be deduced from the variation of the partial derivative of a starscalar. Before doing so we want to introduce a derivative operator $\partial$ that will become very handy. Recall the product rule for the partial derivative (3.14)

$$
\partial_{I}(f \star g)=\partial_{I} f \star g+f \star \partial_{I} g-F_{I}^{J K} \partial_{J} f \star \partial_{K} g .
$$

Multiplying this relation by $d x^{I}$ and interchanging $d x^{I}$ with $f$ using (3.11), one obtains

$$
\begin{aligned}
d x^{I} \star \partial_{I}(f \star g)= & d x^{I} \star \partial_{I} f \star g+d x^{I} \star f \star \partial_{I} g-F^{I J K} d x_{I} \star \partial_{J} f \star \partial_{K} g \\
= & d x^{I} \star \partial_{I} f \star g+f \star d x^{I} \star \partial_{I} g-F^{I J K} d x_{K} \star \partial_{J} f \star \partial_{I} g \\
& -F^{I J K} d x_{I} \star \partial_{J} f \star \partial_{K} g \\
= & d x^{I} \star \partial_{I} f \star g+f \star d x^{I} \star \partial_{I} g .
\end{aligned}
$$

We observe that the correction terms have canceled and we are left with the usual Leibniz rule for the combination $d x^{I} \star \partial_{I}$. It is therefore suggestive to introduce the derivative operator

$$
\partial:=d x^{I} \star \partial_{I} .
$$

$\partial$ raises the degree by one but since we do not assume antisymmetrization $\partial$ is not the exterior derivative $d$. Using $\partial$, (4.12) turns into the usual Leibniz rule

$$
\partial(f \star g)=\partial f \star g+f \star \partial g .
$$


Therefore $\partial$ commutes with the star-product and also the $\mathcal{R}$-matrix. This will simplify many computations from now on.

Let us come back to the variation of the partial derivative of a scalar. Having $\partial$ at hand, we instead compute the variation of the one-form $\partial f=d x^{I} \star \partial_{I} f$. Only the use of $\partial$ allows us to interchange $\delta_{\xi}^{\star}$ with $\partial$ without producing extra terms. We get

$$
\begin{aligned}
\delta_{\xi}^{\star} \partial f & =\partial \delta_{\xi}^{\star} f \\
& =\partial\left(\xi^{I} \star \partial_{I} f\right)=\partial \xi^{I} \star \partial_{I} f+\xi^{I} \star \partial_{I} \partial f,
\end{aligned}
$$

from which we deduce for a form $\omega=d x^{I} \star \omega_{I}$

$$
\mathcal{L}_{\xi}^{*} \omega=\xi^{I} \star \partial_{I} \omega+\partial \xi^{J} \star \omega_{J}
$$

or in components

$$
\mathcal{L}_{\xi}^{*} \omega_{I}=\xi^{J} \star \partial_{J} \omega_{I}+\partial_{I} \xi^{J} \star \omega_{J}
$$

We explicitly checked that the star-Lie-derivatives of vectors (4.6) and forms (4.17) are compatible with the contraction. Therefore a contraction between a vector $V$ and a form $\omega$ indeed transforms as a scalar when computing

$$
\mathcal{L}_{\xi}^{*}\left(V^{I} \star \omega_{I}\right)=\left.\mathcal{L}_{\xi}^{*} V^{I} \star \omega_{I}\right|_{\bar{\phi}}+\left.\overline{\mathcal{R}}\left(V^{I}\right) \star \mathcal{L}_{\overline{\mathcal{R}}(\xi)}^{*} \omega_{I}\right|_{\phi^{2}} .
$$

\subsection{Tensor product}

Let us now define and investigate the notion of a $\star$-tensor product in more detail. In order for tensor products to behave covariantly under star-diffeomorphisms we define $\otimes_{\star}:=$ $\otimes \circ \mathcal{F}^{-1}$ similar to the definition of the commutator and the star-product. Then the Leibniz rule holds

$$
\mathcal{L}_{\xi}^{*}\left(V \otimes_{\star} W\right)=\left.\mathcal{L}_{\xi}^{*} V \otimes_{\star} W\right|_{\bar{\phi}}+\left.\overline{\mathcal{R}}(V) \otimes_{\star} \mathcal{L}_{\overline{\mathcal{R}}(\xi)}^{*} W\right|_{\phi^{2}} .
$$

To apply this rule to an arbitrary element for instance $T \in T M \otimes_{\star} T M$ one might need a split

$$
T=A_{\alpha} \otimes_{\star} B^{\alpha} \in T M \otimes_{\star} T M
$$

with an internal summation over $\alpha$ and $A_{\alpha}=A_{\alpha}^{I} \star \partial_{I} \in T M$ and $B^{\alpha}=B^{\alpha I} \star \partial_{I} \in T M$. The transpose of the above $T$ is defined as $T^{T}=\overline{\mathcal{R}}\left(B^{\alpha}\right) \otimes_{\star} \overline{\mathcal{R}}\left(A_{\alpha}\right)$. When shifting the basis vectors of $A$ and $B$ to the right with (3.10)

$$
\begin{aligned}
T & =A_{\alpha}^{I} \star \partial_{I} \otimes_{\star} B^{\alpha J} \star \partial_{J} \\
& =\left(A_{\alpha}^{I} \star B^{\alpha J}-F^{M N J} A_{\alpha M} \star \partial_{N} B^{\alpha I}\right) \star\left(\partial_{I} \otimes_{\star} \partial_{J}\right)
\end{aligned}
$$

and using (3.18), we find that the transpose $T^{T}$ only interchanges the indices due to nice cancellations

$$
\begin{aligned}
T^{T} & =\overline{\mathcal{R}}\left(B^{\alpha I} \star \partial_{I}\right) \otimes_{\star} \overline{\mathcal{R}}\left(A_{\alpha}^{J} \star \partial_{J}\right) \\
& =\left(A_{\alpha}^{J} \star B^{\alpha I}-F^{M N I} A_{\alpha M} \star \partial_{N} B^{\alpha J}\right) \star\left(\partial_{I} \otimes_{\star} \partial_{J}\right) .
\end{aligned}
$$


Therefore $\mathcal{R}$-symmetric and $\mathcal{R}$-antisymmetric tensors still correspond to symmetric and antisymmetric matrices.

The contraction of tensor products is done by multiplying the forms and vectors standing next to each other with (3.20). Most easily this can be done by bringing the basis vectors and forms to the middle as in (4.21) and eventually applying

$$
\left\langle\partial_{I} \otimes_{\star} \partial_{J}, d x^{A} \otimes_{\star} d x^{B}\right\rangle_{\star}=\delta_{I}^{B} \delta_{J}^{A} .
$$

For $A \otimes_{\star} B \in T M^{2}$ and $\omega \otimes_{\star} \alpha \in T^{*} M^{2}$ we get

$$
\begin{aligned}
\left\langle A \otimes_{\star} B, \omega \otimes_{\star} \alpha\right\rangle_{\star}= & \left(A^{I} \star B^{J}\right) \star\left(\omega_{J} \star \alpha_{I}\right)-F^{I J K}\left(A_{I} \star \partial_{J} B^{M}\right) \star\left(\omega_{M} \star \alpha_{K}\right) \\
& -F^{I J K}\left(A_{I} \star B^{M}\right) \star\left(\partial_{J} \omega_{M} \star \alpha_{K}\right) .
\end{aligned}
$$

With (4.21) and (4.22) and their analogue for forms one can show the transposition symmetry

$$
\left\langle A \otimes_{\star} B, \omega \otimes_{\star} \alpha\right\rangle_{\star}=\left\langle\overline{\mathcal{R}}(B) \otimes_{\star} \overline{\mathcal{R}}(A), \overline{\mathcal{R}}(\alpha) \otimes_{\star} \overline{\mathcal{R}}(\omega)\right\rangle_{\star} .
$$

The generalization to higher rank tensors is straightforward.

Antisymmetric $p$-forms $\omega \in \wedge_{\star}^{p} T^{*} M$ are defined as usual. Of course, here one requires $\mathcal{R}$-antisymmetry and adjusts the star-wedge product to $\wedge_{\star}=\wedge \circ \mathcal{F}^{-1}$. Considering, for instance, the star-wedge product of two one-forms one finds

$$
\omega \wedge_{\star} \alpha=\omega \otimes_{\star} \alpha-\overline{\mathcal{R}}(\alpha) \otimes_{\star} \overline{\mathcal{R}}(\omega)
$$

which is clearly $\mathcal{R}$-antisymmetric. From (4.25) we see that the star-wedge product projects out the antisymmetric part

$$
\left\langle A \otimes_{\star} B, \omega \wedge_{\star} \alpha\right\rangle_{\star}=\left\langle A \otimes_{\star} B, \omega \otimes_{\star} \alpha\right\rangle_{\star}-\left\langle\overline{\mathcal{R}}(B) \otimes_{\star} \overline{\mathcal{R}}(A), \omega \otimes_{\star} \alpha\right\rangle_{\star}
$$

so that one could also define forms by the antisymmetry of their action onto vectors. The exterior derivative is the antisymmetrized partial derivative $d=\partial^{\wedge_{\star}}=d x^{I} \wedge_{\star} \partial_{I}$. Inherited from $\partial$ and (4.14) the exterior derivative $d$ is invariant under the $\mathcal{R}$-matrix or

$$
d\left(\omega \otimes_{\star} \alpha\right)=d \omega \otimes_{\star} \alpha+\omega \otimes_{\star} d \alpha .
$$

This can also be explained from the fact that the star-exterior derivative is the usual exterior derivative, as $d=d x^{I} \wedge_{\star} \partial_{I}=d x^{I} \wedge \partial_{I}$. Since the star-product acts with usual Liederivatives $\mathcal{L}$ satisfying $[\mathcal{L}, d]=0$, the exterior derivative commutes with the star-product and therefore also with $\mathcal{R}$. This was already observed in [28].

\subsection{Comment on $O(D, D)$ metric}

Since we are using a doubled formalism with doubled coordinates $X_{I}=\left(p_{i}, x_{i}\right)$ and doubled vector fields $V^{I}=\left(V^{i}, \tilde{V}_{i}\right)$, one might wonder what the relation to DFT is. The main difference is that we are not dealing here with an $O(D, D)$ covariant formalism. This is reflected in the fact that instead of generalized diffeomorphisms like in DFT we are dealing with only double dimensional ordinary (star-)diffeomorphisms. One could be tempted to define an $O(D, D)$ metric between two vectors via

$$
V^{I} \star W_{I}=V^{i} \star \tilde{W}_{i}+\tilde{V}_{i} \star W^{i} .
$$

However, this is not a (star-)scalar under (star-)diffeomorphisms. 


\section{$5 \quad$ Nonassociative differential geometry}

In this section we continue developing the basic notions of a nonassociative differential geometry. We will discuss star-connections, its torsion and curvature tensors.

\subsection{Covariant derivatives}

The next step is to define a covariant derivative. As we will see, in the nonassociative case there exist two consistent notions of a covariant derivative, where one acts from the left and the other one from the right.

First, we compute the anomalous variation $\Delta_{\xi}^{\star}:=\delta_{\xi}^{\star}-\mathcal{L}_{\xi}^{\star}$ of the derivative $\partial \omega$ of a star-covector $^{4}$

$$
\Delta_{\xi}^{\star} \partial \omega=\partial \partial \xi^{J} \star \omega_{J}
$$

As usual we introduce a Christoffel-symbol $\Gamma$ which we can always write as

$$
\Gamma=d x^{I} \star d x^{J} \star \Gamma_{I J}{ }^{K} \star \partial_{K}
$$

by commuting the basis forms and vectors through with (3.10) and (3.11). Now we can form the operator $\nabla=\partial-\Gamma$ where $\Gamma$ acts with a star-contraction. Dealing with a noncommutative star-product we can either let $\nabla$ act from the left or from the right to form the covariant derivative. Due to (4.14) this corresponds to the ambiguity from which side we want to multiply $\Gamma$

$$
\begin{aligned}
\vec{\nabla} \omega & =\nabla(\omega)=\partial \omega-\Gamma \star \omega \\
\text { or } \quad \overleftarrow{\nabla} \omega & =(\omega) \nabla=\partial \omega-\omega \star \Gamma
\end{aligned}
$$

For $\vec{\nabla}$ the star-contraction in the second term is especially simple giving $\Gamma^{K} \star \omega_{K}$ while for $\overleftarrow{\nabla}$ we need an $\mathcal{R}$-matrix. Moreover the right-linearity of $\vec{\nabla}$ is reminiscent of the rightlinearity of $\omega=d x^{I} \star \omega_{I}$. Nonetheless both covariant derivatives are completely consistent. Computing the anomalous variations $\Delta_{\xi}^{\star}:=\delta_{\xi}^{\star}-\mathcal{L}_{\xi}^{\star}$ of the second terms, we find that both choices correctly compensate the anomalous term in (5.1) if $\Delta_{\xi}^{\star} \Gamma=\partial \partial \xi$

$$
\begin{aligned}
\Delta_{\xi}^{\star}(\Gamma \star \omega) & =\Delta_{\xi}^{\star} \Gamma \star \omega=\partial \partial \xi \star \omega \\
\Delta_{\xi}(\omega \star \Gamma) & =\overline{\mathcal{R}}(\omega) \star \Delta_{\overline{\mathcal{R}}(\xi)} \Gamma=\overline{\mathcal{R}}(\omega) \star \partial \partial \overline{\mathcal{R}}(\xi) \\
& =\overline{\mathcal{R}}(\omega) \star \overline{\mathcal{R}}(\partial \partial \xi)=\partial \partial \xi \star \omega .
\end{aligned}
$$

In the second line we used that the $\mathcal{R}$-matrix commutes with $\partial$ according to (4.14).

Let us mention that from a more axiomatic viewpoint both choices are meaningful. Indeed, in mathematics one defines the covariant derivative as a map $T^{*} M \rightarrow T^{*} M \otimes_{\star} T^{*} M$ obeying the Leibniz rule

$$
\nabla\left(\omega \otimes_{\star} \alpha\right)=\left.\nabla \omega \otimes_{\star} \alpha\right|_{\bar{\phi}}+\left.\overline{\mathcal{R}}(\omega) \star \overline{\mathcal{R}}(\nabla) \alpha\right|_{\phi^{2}} .
$$

\footnotetext{
${ }^{4}$ Here, $\partial$ means the one-form defined in (4.13).
} 
Taking into account that $\nabla f=\partial f$ for scalars, for $\vec{\nabla}$ we deduce

$$
\begin{aligned}
\vec{\nabla} \omega & =\nabla\left(d x^{I} \star \omega_{I}\right)=\left.\nabla\left(d x^{I}\right) \star \omega_{I}\right|_{\bar{\phi}}+\overline{\mathcal{R}}\left(d x^{I}\right) \star \overline{\mathcal{R}}(\partial) \omega_{I} \\
& =\partial \omega-\Gamma \star \omega,
\end{aligned}
$$

while for $\overleftarrow{\nabla}$ we find

$$
\begin{aligned}
\overleftarrow{\nabla} \omega & =\left(d x^{I} \star \omega_{I}\right) \nabla=\left(d x^{I}\right) \overline{\mathcal{R}}(\nabla) \star \overline{\mathcal{R}}\left(\omega_{I}\right)+d x^{I} \star \partial \omega_{I} \\
& =\partial \omega-\omega \star \Gamma
\end{aligned}
$$

So far we defined a covariant derivative acting on covectors. The same procedure can be repeated for vectors without obstructions. We find

$$
\Delta_{\xi}^{\star} \partial V=-\overline{\mathcal{R}}(V) \star \partial \partial \overline{\mathcal{R}}(\xi) .
$$

Along the lines of (5.4), the anomalous transformation $\overline{\mathcal{R}}(V) \star \partial \partial \overline{\mathcal{R}}(\xi)=\partial \partial \xi \star V$ can again be compensated by $\overleftarrow{\nabla}$ and $\vec{\nabla}$ by only changing the overall sign in front of $\Gamma$. This is also consistent with the more axiomatic viewpoint. Therefore, we again have two consistent covariant derivatives

$$
\begin{aligned}
\vec{\nabla} V & =\nabla(V)=\partial V+\Gamma \star V \\
\text { and } \quad \overleftarrow{\nabla} V & =(V) \nabla=\partial V+V \star \Gamma .
\end{aligned}
$$

In contrast to covectors, where $\vec{\nabla} \omega$ was especially simple, now $\overleftarrow{\nabla} V$ becomes simple. Since the basis vectors of $V$ and $\Gamma$ are next to each other, the contraction in the second term simply gives $V^{I} \star \Gamma_{I}$. In addition, the left-linearity of vectors is similar to the left-linearity of $\overleftarrow{\nabla}$.

All this suggests that expressions simplify if we use $\vec{\nabla}$ for covectors and $\overleftarrow{\nabla}$ for vectors. This convention is compatible with the contraction if, similar to $\partial$, the covariant derivative acts without an $\mathcal{R}$-matrix on products ${ }^{5}$

$$
\begin{aligned}
\left.\overleftarrow{\nabla} V \star \omega\right|_{\bar{\phi}}+\left.V \star \vec{\nabla} \omega\right|_{\phi^{2}} & =\partial(V \star \omega)+\left.(V \star \Gamma) \star \omega\right|_{\phi}-\left.V \star(\Gamma \star \omega)\right|_{\phi^{2}} \\
& =\partial(V \star \omega) .
\end{aligned}
$$

Directional covariant derivative. At last we define the directional covariant derivative of a vector $Y=Y^{I} \star \partial_{I}$ simply by multiplication with the directional vector $X=X^{I} \star \partial_{I}$. We can multiply $X$ either from the left or from the right onto $\overleftarrow{\nabla}$ or $\vec{\nabla}$ and therefore have in total four different conventions. Two choices place $X$ and $Y$ next to each other while the other two separate $X$ and $Y$ by a $\Gamma$. To define a star-torsion we will soon see that we better place $X$ and $Y$ together.

To make the contraction in the second term as easy as possible, we define the directional derivative along $X$ of a vector $Y$ as

$$
\begin{aligned}
\nabla_{X} Y:=\overleftarrow{\nabla}_{\vec{X}} Y: & =\left.X \star \overleftarrow{\nabla} Y\right|_{\phi}=X \star \partial Y+\left(X \otimes_{\star} Y\right) \star \Gamma \\
& =X \star \partial Y+\left\langle X \otimes_{\star} Y, \Gamma\right\rangle_{\star}
\end{aligned}
$$

\footnotetext{
${ }^{5}$ Using the same convention for the covariant derivative on covectors and vectors, compatibility with the contraction is also satisfied, but now explicit $\mathcal{R}$-matrices appear

$$
\begin{aligned}
& \vec{\nabla}(V \star \omega)=\left.\vec{\nabla} V \star \omega\right|_{\bar{\phi}}+\left.\overline{\mathcal{R}}(V) \star(\overline{\mathcal{R}}(\vec{\nabla}) \omega)\right|_{\phi^{2}}=\partial(V \star \omega), \\
& \overleftarrow{\nabla}(V \star \omega)=\left.V \star \omega \bar{\nabla}\right|_{\phi}+\left.V \overline{\mathcal{R}}(\overleftarrow{\nabla}) \star \overline{\mathcal{R}}(\omega)\right|_{\bar{\phi}^{2}}=\partial(V \star \omega) .
\end{aligned}
$$
}


where the associator was inserted for convenience. Spelling out the contraction between $X \otimes_{\star} Y$ and $\Gamma$ according to (4.21) reveals a correction term

$$
\begin{aligned}
\nabla_{X} Y= & X^{I} \star \partial_{I} Y^{J} \star \partial_{J}+\left(X^{I} \star Y^{J}\right) \star \Gamma_{I J}{ }^{K} \star \partial_{K} \\
& -F^{M N J}\left(X_{M} \star \partial_{N} Y^{I}\right) \star \Gamma_{I J}{ }^{K} \star \partial_{K} .
\end{aligned}
$$

Recalling (4.22) we find

$$
\begin{aligned}
\nabla_{\overline{\mathcal{R}}(Y)} \overline{\mathcal{R}}(X)= & \overline{\mathcal{R}}(Y)^{I} \star \partial_{I} \overline{\mathcal{R}}(X)^{J} \star \partial_{J}+\left(X^{J} \star Y^{I}\right) \star \Gamma_{I J}{ }^{K} \star \partial_{K} \\
& -F^{M N I}\left(X_{M} \star \partial_{N} Y^{J}\right) \star \Gamma_{I J}{ }^{K} \star \partial_{K} .
\end{aligned}
$$

This is very useful when computing the torsion in the next paragraph.

As a comment, please note that, in the noncommutative though still associative framework of [28], the convention $\nabla_{X} Y=\overleftarrow{\nabla}_{\vec{X}} Y$ is used, as well. ${ }^{6}$

\subsection{Torsion}

We define the star-torsion two-form as usual as the antisymmetrized covariant derivative $\vec{\nabla}^{\wedge \star}$ of the frame $d x^{I}$. As we are in a holonomic frame we find

$$
T^{K}=\vec{\nabla}^{\wedge \star} d x^{K}=\Gamma^{K}=\left(d x^{I} \wedge_{\star} d x^{J}\right) \star \Gamma_{I J}{ }^{K} .
$$

Setting this to zero means

$$
\Gamma_{[I J]}^{K}=0 .
$$

We now want to reproduce the same result from an analogue of the familiar definition $T=\nabla_{X} Y-\nabla_{Y} X-[X, Y]$ with an appropriate insertion of $\mathcal{R}$ matrices. Note that here, for the directional covariant derivative, we used the convention (5.11). We contract $T^{K}$ with vectors $X$ and $Y$ and apply (4.27) to turn the $\wedge_{\star}$ into an antisymmetrization of $X$ and $Y$

$$
\begin{aligned}
T(X, Y): & =\left\langle X \otimes_{\star} Y, T^{K} \star \partial_{K}\right\rangle_{\star} \\
& =\left\langle X \otimes_{\star} Y, d x^{I} \wedge_{\star} d x^{J} \star \Gamma_{I J}{ }^{K} \star \partial_{K}\right\rangle_{\star} \\
& =\left\langle X \otimes_{\star} Y-\overline{\mathcal{R}}(Y) \otimes_{\star} \overline{\mathcal{R}}(X), d x^{I} \otimes_{\star} d x^{J} \star \Gamma_{I J}{ }^{K} \star \partial_{K}\right\rangle_{\star}
\end{aligned}
$$

In the second line we identify the $\Gamma$ terms from the covariant derivative (5.11). By adding and subtracting the missing terms $X \star \partial Y=X(Y)$ we can reproduce the torsion via

$$
T(X, Y)=\nabla_{X} Y-\nabla_{\overline{\mathcal{R}}(Y)} \overline{\mathcal{R}}(X)-[X, Y]_{\star} .
$$

At this point we need a convention where $X$ and $Y$ are next to each other, as otherwise $X$ and $Y$ would be separated by $\Gamma$. An explicit computation of the torsion by inserting (5.12) and (5.13) gives

$$
T(X, Y)=\left(X^{I} \star Y^{J}+F^{I M N} X_{M} \star \partial_{N} Y^{J}\right) \star\left(\Gamma_{I J}-\Gamma_{J I}\right) .
$$

The torsion tensor for basis vectors comes out as

$$
\left\langle T\left(\partial_{I}, \partial_{J}\right), d x^{K}\right\rangle_{\star}=\Gamma_{I J}^{K}-\Gamma_{J I}^{K} .
$$

\footnotetext{
${ }^{6}$ In [28] they demand $\nabla_{X}(f \star Y)=\overline{\mathcal{R}}(f) \star \nabla_{\overline{\mathcal{R}}(X)} Y$, which is only possible if $X$ and $Y$ are placed next to each other. Also in equation 5.4 of [28], the left action $\overleftarrow{\nabla}$ is used.
} 


\subsection{Riemann and Ricci tensor}

In an analogous manner we can proceed to derive a star-generalization of the Riemann curvature. The curvature two-form can be defined as the exterior covariant derivative of the connection $\Gamma=d x^{K} \otimes_{\star} d x^{I} \star \Gamma_{K I}{ }^{L} \star \partial_{L}$ which we consider as a matrix-valued one form $\Gamma_{K}^{L}:=d x^{I} \star \Gamma_{K I}{ }^{L}$

$$
R_{K}^{L}=\nabla^{\wedge} \Gamma_{K}^{L}=d \Gamma_{K}^{L}-\Gamma_{K}^{P} \wedge_{\star} \Gamma_{P}^{L} .
$$

We can contract the matrix indices with basis vectors and write

$$
R=d x^{K} \star R_{K}^{L} \star \partial_{L}=d \Gamma-\Gamma \wedge_{\star} \Gamma
$$

where $d$ is meant to act on the one form part of $\Gamma$. Using (5.21), tensoriality of $R$ can be readily checked using the anomalous transformation $\Delta_{\xi}^{\star} \Gamma=\partial \partial \xi$, the nilpotency of $d$ in $d \partial \xi=d d \xi=0$ and the $\mathcal{R}$-antisymmetry (4.26) of $\wedge_{\star}$.

As the curvature $R$ contains three basis one-forms, we can contract it with three vectors

$$
\begin{aligned}
R(X, Y, Z): & =\left\langle\left(X \otimes_{\star} Y\right) \otimes_{\star} Z, R\right\rangle_{\star} \\
& =\left\langle\left(X \otimes_{\star} Y\right)^{\phi},\left(Z^{K \phi} \star R_{K}{ }^{L \phi} \star \partial_{L}\right)\right\rangle_{\star} .
\end{aligned}
$$

Similar to the torsion, one expects this to match the alternative definition

$$
R(X, Y, Z)=-\left(\left(\nabla_{X} \bullet \nabla_{Y}\right) Z-\left(\nabla_{\overline{\mathcal{R}}(Y)} \bullet \nabla_{\overline{\mathcal{R}}(X)}\right) Z-\nabla_{[X, Y]_{\star}} Z\right) .
$$

The minus sign in this definition is just a convention needed to match both definitions. In order to evaluate (5.23), we need to clarify the meaning of the composition $\bullet$ for the directional covariant derivatives.

Having a closer look at (5.11), one realizes that $\nabla_{X} Y$ consists actually of two consecutive operations: First the action of $\overleftarrow{\nabla}=\partial+\Gamma$ from the right and second the contraction with $X$ from the left, denoted in the following by $i_{X}$. Next, we apply an associator to bracket $X$ and $Y$ together. Following this prescription, we can write

$$
\begin{aligned}
\nabla_{X} Y: & =\left(i_{X}(Y)\right) \overleftarrow{\nabla}=i_{X}^{\phi}\left(Y^{\phi} \overleftarrow{\nabla}^{\phi}\right) \\
& =i_{X} \partial Y+i_{X}^{\phi}\left(Y^{\phi} \star \Gamma^{\phi}\right)=X \star \partial Y+\left(X \otimes_{\star} Y\right) \star \Gamma
\end{aligned}
$$

The composition • in (5.23) must then be understood as the composition of both the left and right acting operators

$$
\left(\nabla_{X} \bullet \nabla_{Y}\right) Z=\left[\left(i_{X} \bullet i_{Y}\right)(Z)\right](\overleftarrow{\nabla} \bullet \overleftarrow{\nabla})
$$

In appendix B, we show explicitly the equivalency of (5.22) and (5.23), when we evaluate these four operations in the appropriate order.

To compute the components of the star-Riemann tensor, we need to shift all basis vectors in (5.22) into the middle with (3.11) and (3.10)

$$
\begin{aligned}
R=d x^{K} \otimes_{\star} d x^{I} \wedge_{\star} d x^{J} & \\
\star & {\left[\partial_{I} \Gamma_{K J}{ }^{L}-\Gamma_{K I}^{P} \star \Gamma_{P J}{ }^{L}-F_{I}^{A B} \partial_{A} \Gamma_{K J}{ }^{P} \star \Gamma_{P B}{ }^{L}\right] \star \partial_{L} }
\end{aligned}
$$


and

$$
\begin{aligned}
\left(X \otimes_{\star} Y\right) \otimes_{\star} Z=[ & \left(X^{J} \star Y^{I}\right) \star Z^{K} \\
& -F^{A B J} X_{A}^{\phi} \star \partial_{B}\left(Y^{I \phi} \star Z^{K \phi}\right)-F^{A B I} \star\left(X^{J} \star Y_{A}\right) \star \partial_{B} Z^{K} \\
& \left.-F^{A B J} F^{C D I} X_{A}^{\phi} \star \partial_{B}\left(Y_{C}^{\phi} \star \partial_{D} Z^{K \phi}\right)\right] \star \partial_{J} \otimes_{\star} \partial_{I} \otimes_{\star} \partial_{K} .
\end{aligned}
$$

We denoted the indices in such a way that $R(X, Y, Z)$ can be directly read off by starmultiplying the [...] brackets from (5.26) and (5.27). The components of the star-curvature thus contain a correction term proportional to the $R$-flux

$$
\begin{aligned}
R_{I J K}{ }^{L} & :=\left\langle R\left(\partial_{I}, \partial_{J}, \partial_{K}\right), d x^{L}\right\rangle_{\star} \\
& =2 \partial_{[\underline{I}} \Gamma_{K \underline{J}]}{ }^{L}-2 \Gamma_{K\left[\underline{I}^{M}\right.}{ }^{M} \Gamma_{M \underline{J}]^{L}}-2 F_{[\underline{I}}^{A B} \partial_{A} \Gamma_{K \underline{J}]^{M}}{ }^{M} \Gamma_{M B}{ }^{L} .
\end{aligned}
$$

For a torsion-free connection, one can directly check that the first Bianchi identity

$$
R_{I J K}{ }^{L}+R_{K I J}{ }^{L}+R_{J K I}^{L}=0
$$

is still satisfied. The second Bianchi-identity receives a correction that, at leading order in the flux $F$, is related to the associator of three connections

$$
\frac{1}{2} \nabla_{[\underline{I}} R_{\underline{J K}] M}{ }^{N}=\left[\left(\Gamma_{[\underline{I} M}{ }^{A} \star \Gamma_{\underline{J}} A^{B}\right) \star \Gamma_{\underline{K}] B}{ }^{N}-\Gamma_{\left[\underline{I} M^{A} \star\right.}^{A}\left(\Gamma_{\underline{J}} A^{B} \star \Gamma_{\underline{K}] B}{ }^{N}\right)\right]+O(F) .
$$

Notice that we need to use the convention $\vec{\nabla}$ for covectors and $\overleftarrow{\nabla}$ for vectors to cancel the terms of the form $\sim \partial \Gamma \star \Gamma$ and $\sim \Gamma \star \partial \Gamma$. As usual, the Ricci tensor is the trace of the Riemann tensor

$$
\operatorname{Ric}(Y, Z):=\left\langle R\left(\partial_{I}, Y, Z\right), d x^{I}\right\rangle_{\star}
$$

\section{Features of a star-metric}

In gravity the fundamental field is not a connection but a metric $G \in T^{*} M \otimes T^{*} M$ that allows to measure distances on the manifold. Given a metric one then defines the Levi-Civita connection to be the torision-free connection that warrants a covariantly constant metric. In this section we will see that the generalization of this procedure to the nonassociative case appears to be less straightforward. Since so far we did not find a fully satisfying resolution of the encountered obstacles, this section should be understood as a first approach to this problem. In most parts of this section, we restrict our considerations to star-tensors which depend only on configuration space.

Before we move on, let us recall that in differential geometry the metric is used in two ways. First it provides a scalar product between two vectors from the tangent space, i.e.

$$
(v, w)^{g}=g_{i j} v^{i} w^{j} .
$$

Second it is considered to be a duality map $\mathcal{G}: T M \rightarrow T^{*} M$ that allows to lower indices

$$
\mathcal{G}(v)_{j}=g_{i j} v^{j} .
$$

The scalar product in $(6.1)$ is then identical to $(v, w)^{\mathcal{G}}=\langle v, \mathcal{G}(w)\rangle$. Moreover, the inverse metric can be used to raise indices and of course one has

$$
\mathcal{G}^{-1}(\mathcal{G}(v))=v, \quad g^{i j}\left(g_{j k} v^{k}\right)=v^{i} .
$$




\subsection{Metric}

We introduce a star-metric $G$ as an $\mathcal{R}$-symmetric element in $T^{*} \mathcal{M} \otimes_{\star} T^{*} \mathcal{M}$, i.e. it satisfies

$$
G(X, Y)=G(\overline{\mathcal{R}}(Y), \overline{\mathcal{R}}(X))
$$

with

$$
G(X, Y):=\left\langle X \otimes_{\star} Y,\left(d x^{I} \otimes_{\star} d x^{J}\right) \star g_{I J}\right\rangle_{\star} .
$$

Recalling (4.21) and (4.22) one obtains

$$
\begin{aligned}
0 & =G(X, Y)-G(\overline{\mathcal{R}}(Y), \overline{\mathcal{R}}(X)) \\
& =\left(X^{I} \star Y^{J}+F^{I M N} X_{M} \star \partial_{N} Y^{J}\right) \star\left(g_{I J}-g_{J I}\right)
\end{aligned}
$$

so that $g$ must be symmetric in the usual sense $g_{I J}=g_{J I}$. Turning this around, every symmetric tensor gives rise to a star-metric.

$\mathbf{R}$-symmetric scalar product. The first definition of the scalar product is

$$
(V, W)_{\star}^{g}:=\left\langle\left(V \otimes_{\star} W\right), g\right\rangle_{\star} .
$$

With (4.25) the $\mathcal{R}$-symmetry of $g$ translates into the $\mathcal{R}$ symmetry between the vectors

$$
\left\langle\left(V \otimes_{\star} W\right), g\right\rangle_{\star}=\left\langle\overline{\mathcal{R}}(W) \otimes_{\star} \overline{\mathcal{R}}(V), g\right\rangle_{\star} .
$$

For the easiest example where $v=v \star \partial_{i}$ and $w=w \star \partial_{i}$, this scalar product is

$$
(v, w)_{\star}^{g}:=\left(v^{i} \star w^{j}\right) \star g_{i j} .
$$

The metric as a star-duality map. Similar to the usual case, we can also interprete the metric $g$ as the duality map $\mathcal{G}: T \mathcal{M} \stackrel{g}{\rightarrow} T^{*} \mathcal{M}$ acting through $\mathcal{G}(W)=\langle W, g\rangle_{\star} \in T^{*} \mathcal{M}$. Let us again only consider the easiest example $v=v \star \partial_{i}$ and $w=w \star \partial_{i}$. When we compute $\langle v, \mathcal{G}(w)\rangle_{\star}$ one finds

$$
(v, w)^{\mathcal{G}}=v^{i} \star\left(w^{j} \star g_{i j}\right)
$$

which is not the same as the star-scalar product (6.9). In fact the two are related by applying an associator

$$
(v, w)_{\star}^{g}=\left.(v, w)^{\mathcal{G}}\right|_{\phi} .
$$

As a consequence of the appearing associator, $(v, w)^{\mathcal{G}}$ is not $\mathcal{R}$-symmetric.

A second deviation from the usual case appears when one considers the inverse of the star-duality map $\mathcal{G}^{-1}: T^{*} \mathcal{M} \stackrel{g^{-1 \star}}{\longrightarrow} T \mathcal{M}$, which should satisfy $\mathcal{G}^{-1}(\mathcal{G}(v))=v$ for all $v \in T \mathcal{M}$. In components this reads

$$
\left(v^{k} \star g_{k j}\right) \star\left(g^{-1 \star}\right)^{j i}=v^{i}
$$

and deviates from the usual case in the sense that, due to nonassociativity, an inverse satisfying

$$
g_{i j} \star\left(g^{-1 \star}\right)^{j k}=\delta_{i}^{k}
$$

does not satisfy (6.12). In the noncommutative but associative case, a construction of a general star-inverse in the sense of (6.13) was provided in [27, 33]. 


\subsection{The star-inverse}

It is clear that in order to proceed along the usual lines, one needs a star-inverse of the metric. Recall that the inverse of the metric appears explicitly in the Levi-Civita connection and in the definition of the Ricci-scalar. In general it is unclear whether a solution to (6.13) exists. However, as emphasized in the introduction, from the string theory viewpoint, it is actually only up to linear order in $R^{i j k}$ that the star-product is really trustable. Recall that when the nonassociative product was derived in [6], it was done for a flat metric with a constant $R$-flux, which is only a solution of the string equations of motion up to linear order in $R$. In this section, we therefore consider first the construction of the inverse of a scalar and second the construction of an inverse of the star-metric up to linear order in the $R$-flux.

Star inverse of a scalar. Let us consider the simpler question of constructing the starinverse $f^{-1 \star}$ of a scalar $f$, which has to satisfy

$$
f^{-1 \star} \star(f \star g)=g, \quad \forall g
$$

We sort this equation according to the derivatives acting on $g$. At zeroth order in derivatives of $g$ the star-product between $f$ and $g$ becomes a usual multiplication

$$
f \star g=f g+\partial_{I} g \cdot \ldots
$$

When carrying out the remaining star-product in (6.14), since all derivatives act only on $f$, we find at zeroth order in derivatives of $g$

$$
f^{-1 \star} \star(f \star g)=\left(f^{-1 \star} \star f\right) \cdot g+\partial_{I} g \ldots
$$

Since this must be equal to $g$, we conclude that $f^{-1 \star}$ has to satisfy

$$
f^{-1 \star} \star f=1
$$

For general $g$ this is a contradiction to (6.14) unless the associator of $f^{-1 \star}$ and $f$ trivializes, i.e. $\phi\left(f^{-1 \star}, f, \cdot\right)=1$. Of course, we do not expect that this is a generic situation. ${ }^{7}$ However, there exist certain scalars for which the star-inverse can be identified. Consider e.g. the exponentials $f(x)=\exp (i \vec{q} \vec{x})$ from section 2.4. As one can easily show, in this case the $\star$-inverse is simply $f^{-1 \star}(x)=\exp (-i \vec{q} \vec{x})$. Indeed this scalar satisfies

$$
\phi\left(f^{-1 \star}, f, .\right)=1, \quad f^{-1 \star} \star f=f^{-1 \star} \cdot f=1 .
$$

As a matter of fact one can show that, for a map $h \in C^{\infty}(M)$, the star-inverse of $\mathfrak{h}(\vec{x})=$ $h(\vec{q} \vec{x})$ is $\mathfrak{h}^{-1 \star}=1 / h(\vec{q} \vec{x})$.

\footnotetext{
${ }^{7}$ Nonassociative algebras satisfying $\phi(f, f, \cdot)=\phi(\cdot, f, f)=1$ are called alternative. See [34] for a recent discussion in the context of nonassociative star-products. As can be seen from (2.12), for general momentum our star-product is not alternative.
} 
Star-inverse of the metric. Let us now come back to the metric $g_{i j}(x)$ and from now on proceed in linear order in the $R$-flux. At this order, we try to find solutions to

$$
g_{i j} \star g_{R}^{\star-1 j k}=\delta_{i}^{k}+\mathcal{O}\left(R^{2}\right), \quad g_{L}^{\star-1 i j} \star g_{j k}=\delta_{i}^{k}+\mathcal{O}\left(R^{2}\right)
$$

where we distinguished between a right- and a left-inverse. Remarkably, up to linear order, one can explicitly solve these equations

$$
\begin{aligned}
& g_{R}^{\star-1 i j}=g^{i j}-\frac{i l_{s}^{4}}{6 \hbar} R^{a b c} p_{c} g^{i m} \partial_{a} g_{m n} \partial_{b} g^{n j}+\frac{l_{s}^{4}}{12} R^{a b c} \partial_{a} g^{i m} \partial_{b} g_{m n} \partial_{c} g^{n j} \\
& g_{L}^{\star-1 i j}=g^{i j}-\frac{i l_{s}^{4}}{6 \hbar} R^{a b c} p_{c} \partial_{a} g^{i m} \partial_{b} g_{m n} g^{n j}-\frac{l_{s}^{4}}{12} R^{a b c} \partial_{a} g^{i m} \partial_{b} g_{m n} \partial_{c} g^{n j}
\end{aligned}
$$

For these star-inverse metrics we observe:

- They are not symmetric any longer. For their symmetric parts one finds $g_{L / R}^{\star-1(i j)}=g^{i j}$ and the antisymmetric parts are given by the linear corrections in (6.20).

- The star-inverses are momentum dependent, even if the original star-metric was not.

- Taking into account (2.12), one realizes that the left- and the right-inverse differ by an associator.

The latter point is explicitly reflected by expressing (6.20) as

$$
\begin{aligned}
& g_{R}^{\star-1 i j}=2 g^{i j}-g^{i m} \star\left(g_{m n} \star g^{n j}\right), \\
& g_{L}^{\star-1 i j}=2 g^{i j}-\left(g^{i m} \star g_{m n}\right) \star g^{n j} .
\end{aligned}
$$

In this form the inverses are very similar to the inverse metric on the Moyal-Weyl-Plane in [27]. However, this inverse does not satisfy (6.12), as

$$
\left(v^{k} \star g_{k j}\right) \star\left(g_{R}^{-1 \star}\right)^{j i}=v^{i}+\frac{l_{s}^{4}}{6} R^{a b c} \partial_{a} v^{k} \partial_{b} g_{k j} \partial_{c} g^{j i}+\mathcal{O}\left(R^{2}\right),
$$

where the second term is in general not vanishing as $\phi\left(g_{i j}, g^{j k},.\right) \neq 1$. As a consequence, the existence of these star-inverse metrics does not allow us to solve equations involving the metric.

\subsection{Comments on Levi-Civita connection}

In this final section we discuss the consequences of the previous discussion on the construction of a star-Levi-Civita connection. The latter is a torsion-free, metric compatible connection, i.e $\nabla g=0$. In this section, we do not restrict to the space-time components of the star-metric but also consider the momentum components.

In this general case, the condition for the star-inverse of the metric reads

$$
\begin{aligned}
\delta & =g \star g^{\star-1} \\
& =d x^{I} \otimes_{\star} d x^{J} \star g_{I J} \star g^{\star-1 A B} \star \partial_{A} \otimes_{\star} \partial_{B} \\
& =d x^{I} \star\left(g_{I J} \star\left(g^{\star-1}\right)^{J B}+F_{A}{ }^{M N} \partial_{M}\left(g_{I N} \star\left(g^{\star-1}\right)^{A B}\right)\right) \star \partial_{B},
\end{aligned}
$$


where the additional term is there to compensate the shift of the basis vector and no obstacle in finding an inverse. To embed the space-time dynamics into the phase space we make the ansatz ${ }^{8}$

$$
g=d x^{i} \otimes_{\star} d x^{j} \star g_{i j}(x)+\frac{d p_{i}}{i \hbar} \otimes_{\star} \frac{d p_{i}}{i \hbar} \star \eta^{i j} .
$$

From (6.23) we see that we need a compensating term for the shift of basis vectors giving

$$
\begin{aligned}
g_{R}^{\star-1}= & g_{R}^{\star-1 i j} \star \partial_{i} \otimes_{\star} \partial_{j}+\eta_{i j} \star i \hbar \tilde{\partial}_{p}^{i} \otimes_{\star} i \hbar \tilde{\partial}_{p}^{j} \\
& -\frac{l_{s}^{4}}{6} R^{a m n} g^{b i} \star \eta_{a j} \star \partial_{m} g_{b n} \star \partial_{i} \otimes_{\star} \tilde{\partial}_{p}^{j} .
\end{aligned}
$$

Vanishing torsion implies $\Gamma_{[I J]}^{K}=0$ so that, proceeding in the usual way, we arrive at the relation

$$
\begin{aligned}
\left(d x^{I} \otimes_{\star} d x^{J} \otimes_{\star} d x^{K}\right) \star\left[\partial_{I} g_{J K}+\partial_{J} g_{I K}-\partial_{K} g_{I J}\right] & =\left(d x^{I} \otimes_{\star} d x^{J}\right) \star 2 \Gamma_{I J}{ }^{L} \star d x^{K} \star g_{L K} \\
& =2 \Gamma \star g,
\end{aligned}
$$

that needs to solved for $\Gamma$. However, due to the appearing associator in (6.22), this is not solved by $\Gamma=\left(\sum \partial g\right) \star g_{R}^{\star-1}$, where $\sum \partial g=\left(\partial_{I} g_{J K}+\partial_{J} g_{I K}-\partial_{K} g_{I J}\right)$.

We observe that for the Levi-Civita connection, the obstruction arising from (6.22) could in principle be cured by reordering the brackets in the Levi-Civita connection by hand, i.e. by defining its covariant derivative on a covector as

$$
\nabla^{\mathrm{LC}} \omega=\partial \omega-\left(\left(\sum \partial g\right) \circ g_{L}^{\star-1}\right)(\omega)=\partial \omega-\left(\sum \partial g\right) \star\left(g_{L}^{\star-1} \star \omega\right) .
$$

Note that this is just an ad-hoc measure that is not consistent with the $\bullet$-composition introduced in (2.28), which involved an extra application of the associator. Most importantly, as pointed out in [26], when using the $\circ$ composition, one is in general not considering the connection $\Gamma$ as an independent object. ${ }^{9}$

Since this implies a major deviation from the structure introduced so far, the precise justification of such a definition of the connection is beyond the scope of this paper. Nevertheless, we would like to finish our analysis with some comments about the remaining step of defining a star-Einstein Hilbert action.

Final consideration on Einstein-Hilbert action. For defining an Einstein-Hilbert action (up to linear order in $R$ ), one needs a measure $\mu$ that should transform as a starscalar density under star-diffeomorphisms

$$
\delta_{\xi} \mu=\xi^{I} \star \partial_{I} \mu+\left(\partial_{I} \xi^{I}\right) \star \mu .
$$

In this case the Einstein-Hilbert action

$$
S=\int d^{d} x d^{d} p(\mu \star \text { Ric })
$$

\footnotetext{
${ }^{8}$ Considering the metric instead of the vielbein as the fundamental field is also motivated by string theory, where the star-product appears between the vertex operators and therefore between the fluctuations of the metric.

${ }^{9}$ If this would be possible, then $(A \circ B) \star 1=A \star(B \star 1)=A * B$, so that $(A \circ B)=A \star B$, implying $(A \star B) \star C=(A \circ B) \star C=A \star(B \star C)$. This is not satisfied in the nonassociative case [26].
} 
is star-diffeomorphism invariant as

$$
\delta_{\xi} S=\int d^{d} x d^{d} p \partial_{I}\left(\xi^{I} \star(\mu \star \mathrm{Ric})\right) .
$$

We make the usual choice $\mu=\sqrt{g}$ and, in the spirit of the comment in section 2.4, consider it as an elementary object that only depends on $x$.

Since we have the aforementioned bracketing issue in the Levi-Civita connection, in the following we will make some general comments while being agnostic about the it. Now we consider the embedding (6.24) and restrict the action to configuration space via

$$
S=\int d^{d} x d^{d} p \sqrt{g} \star \operatorname{Ric} \star \delta(p) .
$$

Following the discussion in section 2.2, the $\delta(p)$ embeds the configuration space into the phase space as the $p_{0}=0$ leaf. ${ }^{10}$ Let us now analyze the linear terms in $R^{a b c}$. Two terms in the Ricci scalar have the formal structure

$$
S_{1}=\int d^{d} x \sqrt{g} \star g^{-1} \star \partial \Gamma=\int d^{d} x \sqrt{g} \star g^{-1} \star \partial\left(\partial g \star g^{-1}\right) .
$$

where we neither specify the bracketing nor the order and leave it also open whether the left or the right inverse of the metric appears.

In the spirit of the comment from the introduction we want to know whether there exists the possibility that the linear $R$-flux correction is a total derivative(so that the nonassociativity does not leave any trace in the action). There are two sources of linear terms in the $R$-flux:

1. They can appear from the star-product between the objects that only depend on the coordinates $x$, i.e. $\left\{g_{i j}, \sqrt{g},\left(g_{L / R}^{*-1}\right)^{(i j)}\right\}$.

2. The star-inverse has a linear correction $\left(g_{L / R}^{*-1}\right)^{[i j]}$ that depends linearly on $R^{a b c}$ and also on the momentum coordinates $p$.

Terms from category 1 are becoming total derivatives once they are bracketed in the nested way of eq. (2.14). Terms of category 2 can be trivially absent if $\left(g_{L / R}^{*-1}\right)^{[i j]}$ is coupled to symmetrized indices $(i j)$. Moreover, we observe that

$$
\begin{aligned}
\left.\int d^{d} x\left(g_{L}^{*-1}\right)^{[i j]} \star \psi_{i j}\right|_{p_{0}=0} & =\int d^{d} x R^{a b c} \partial_{a} g^{i m} \partial_{b} g_{m n} \partial_{c}\left(g^{n j} \psi_{i j}\right) \\
& =\int d^{d} x \partial_{c}\left(R^{a b c} \partial_{a} g^{i m} \partial_{b} g_{m n} g^{n j} \psi_{i j}\right)
\end{aligned}
$$

so that the linear $R$-correction for a left-placed $g_{L}^{*-1}$ gives a total derivative. Similarly, for a right-placed $g_{R}^{*-1}$ one finds

$$
\left.\int d^{d} x \psi_{i j} \star\left(g_{R}^{*-1}\right)^{[i j]}\right|_{p_{0}=0}=\int d^{d} x \partial_{c}\left(R^{a b c} \psi_{i j} g^{i m} \partial_{a} g_{m n} \partial_{b} g^{n j}\right) .
$$

\footnotetext{
${ }^{10}$ One could also carry out the momentum integral without the $\delta(p)$, as $p$ enters only through the starproduct and therefore linearly. Taking into account that $\int_{\mathbb{R}^{d}} d^{d} p p^{\mu}=0$ one is confined to the $p_{0}=0$ leaf, anyway.
} 
Since in (6.32) there appear only two $g^{*-1}$ factors, there exist an order/bracketing that only gives total derivatives at linear order in $R^{a b c}$.

The remaining terms in the Ricci-scalar are of the schematic form

$$
S_{2}=\int d^{d} x \sqrt{g} \star g^{-1} \star \Gamma \star \Gamma=\int d^{d} x \sqrt{g} \star g^{-1} \star \partial g \star g^{-1} \star \partial g \star g^{-1} .
$$

Here we have three factors of $g^{*-1}$ so that one of them cannot be placed entirely to the left or to the right. A more detailed look at the index structure reveals that some of these terms are not trivially vanishing (i.e. coupling to a symmetrized pair of indices).

Thus, we conclude that, irrespective of the bracketing/ordering, there is no obvious reason why these corrections linear in $R^{a b c}$ should give a total derivative. This could only happen via some cancellations of terms, which however depends on the details of the ordering/bracketing. If linear effects remain, these will be of sixth order in derivatives and are expected to break the usual diffeomorphism symmetry. This makes it questionable whether they have anything to do with string theory.

From the string theory perspective, we recall from [6] that the tri-product was derived for tachyon vertex operators only, while already the definition of a graviton vertex operator in a linear R-flux background was not achieved in a straightforward manner. Therefore, one might be sceptical about the simple appearance of the tri-product between metric factors in the first place. In view of [6], another possibility could be that the metric itself (and not only its star-inverse) receives some order $R$-corrections. Of course, all this is very speculative so that we stop here.

\section{Conclusions}

In this paper, in a step by step procedure we have (re-)derived the salient structure of a nonassociative differential geometry that is based on the nonassociative star-product arising for the closed string moving in a constant nongeometric $R$-flux background. Remarkably, even without associativity is was possible to generalize the notions of diffeomorphisms, tensors, covariant derivatives, torsion and curvature. This was possible, as mathematically one is dealing with still a special way of how associativity is broken, namely that its information is encoded in an $\mathcal{R}$-matrix and an associator $\phi$. Such a structure, namely the differential geometry associated to a quasi-Hopf algebra, was recently developed from a very mathematical and abstract point of view in [30-32]. In an attempt to make these results more accessible to physicists, we tried to motivate and clarify the appearing structure for our concrete $R$-flux example from a bottom up perspective.

As in [30-32], the gravity theory could be well developed up to the point where a metric and its Levi-Civita connection are introduced. We argued that due to the nonassociativity, the star-metric generically does not satisfy the usual relations for pulling up and down indices. Up to linear order in the flux, left/right-inverses of the metric could be identified that however were not symmetric and did not allow a calculus, where equations could be solved. Of course, it could well be that we are missing a resolution of all these problems but it could also indicate that there is something seriously wrong about introducing a metric on such spaces. 
At the end we were pointing out that maybe one needs to define the action of the LeviCivita connection in a different way that employs the o-composition introduced in [26]. Whether this major deviation from the structure introduced before leads to a consistent nonassociative gravity theory remains to be seen. Finally, we were commenting on the construction of a star-Einstein-Hilbert action and generally discussed whether it could be possible that, up to first order in the $R$-flux, all effects of nonassociativity disappear after restricting to the $p_{0}=0$ leaf. Of course this discussion only becomes truly relevant after the issue about the definition of the star-Levi-Civita connection has been resolved.

Let us close by mentioning again that, at the momentary state of affairs, it is an open question whether such a nonassociative gravity theory based on the concept of stardiffeomorphisms has really anything to do with string or double field theory, but it is certainly a viable and interesting possibility that deserves further studies in mathematical physics.

\section{Acknowledgments}

We are grateful to G. Barnes, P. Schupp and R. Szabo for discussions.

\section{A Hopf algebra approach}

Above we derived an adjusted Leibniz rule to make the star-product behave covariantly. Mathematically this can be captured by Hopf algebras. We did not want to present our results in the abstract language of Hopf algebras for readability. Thus instead of being mathematically precise we will only give a short introduction into the topic to understand how the twisted Leibniz rule appears naturally in the context of Hopf algebras. Important for us are now only the multiplication $\mu$ and the coproduct $\Delta$ of the Hopf algebra. As usual the multiplication takes two objects and multiplies them to one. The comultiplication does the opposite. It takes one object and gives out two. Therefore

$$
\begin{gathered}
\mu: H \otimes H \rightarrow H, \\
\Delta: H \rightarrow H \otimes H .
\end{gathered}
$$

The Hopf algebra we are interested in is the universal enveloping algebra of the diffeomorphisms. It consists of what is usually denoted by $\delta_{\xi}$, thus the differential operator that becomes the actual transformation when acting on for instance a scalar $\delta_{\xi} \phi=\xi^{\mu} \partial_{\mu} \phi$. The multiplication is the usual one while the coproduct is

$$
\Delta\left(\delta_{\xi}\right)=\delta_{\xi} \otimes 1+1 \otimes \delta_{\xi} .
$$

Now it is clear that we should interpret the comultiplication $\Delta$ as the Leibniz rule for differentiation. For instance when acting on the product of two scalars we have

$$
\delta_{\xi}(\phi \psi)=\left(\delta_{\xi} \phi\right) \psi+\phi\left(\delta_{\xi} \psi\right)=\mu\left(\delta_{\xi} \phi \otimes \psi+\phi \otimes \delta_{\xi} \psi\right)=\mu \circ \Delta\left(\delta_{\xi}\right)(\phi \otimes \psi) .
$$

The left side can also be written as $\delta_{\xi} \mu(\phi \otimes \psi)$ which can be compared with the right side to

$$
\delta_{\xi} \mu=\mu \circ \Delta\left(\delta_{\xi}\right)
$$


We will now deform our product with a twist $\mathcal{F}$ as above (2.1) to

$$
\mu_{\star}(f \otimes g):=\mu \circ \mathcal{F}^{-1}=f \star g
$$

and demand the product to be compatible with the coproduct. A short calculation gives a twisted Leibniz rule

$$
\begin{aligned}
\delta_{\xi}(\phi \star \psi) & =\delta_{\xi}\left(\mu \circ \mathcal{F}^{-1}(\phi \otimes \psi)\right)=\mu \circ \Delta\left(\delta_{\xi}\right) \circ \mathcal{F}^{-1}(\phi \otimes \psi) \\
& =\mu \circ \mathcal{F}^{-1} \circ \mathcal{F} \circ \Delta\left(\delta_{\xi}\right) \circ \mathcal{F}^{-1}(\phi \otimes \psi) \\
: & =\mu_{\star} \circ \Delta_{\star}\left(\delta_{\xi}\right)(\phi \otimes \psi)
\end{aligned}
$$

where the new coproduct $\Delta_{\star}\left(\delta_{\xi}\right):=\mathcal{F} \Delta\left(\delta_{\xi}\right) \mathcal{F}^{-1}$ dictates the twisted Leibniz rule. As one can show these twisted objects still satisfy the axioms of a Hopf algebra or a generalization called quasi-Hopf algebra. So far we only used the generators $\partial_{\mu}$ for which we can calculate $\Delta_{\star}\left(\partial_{\mu}\right)=\Delta\left(\partial_{\mu}\right)$. In contrast to this we get

$$
\Delta_{\star}\left(\tilde{\partial}_{p}^{\mu}\right)=\Delta\left(\tilde{\partial}_{p}^{\mu}\right)+\frac{i l_{s}^{4}}{6 \hbar} R^{\mu \nu \rho}\left(\partial_{\nu} \otimes \partial_{\rho}\right)
$$

and therefore the additional terms in the Leibniz rule as in (3.14).

\section{B Computing the Riemann tensor}

In this appendix we provide the details on the evaluation of

$$
-R(X, Y, Z)=\left(\nabla_{X} \bullet \nabla_{Y}\right) Z-\left(\nabla_{\overline{\mathcal{R}}(Y)} \bullet \nabla_{\overline{\mathcal{R}}(X)}\right) Z-\nabla_{[X, Y]_{\star}} Z .
$$

As discussed in the main text after (5.23), we need to interpret the $\bullet$ as a composition of left and right actions of the directional covariant derivative

$$
\left(\nabla_{X} \bullet \nabla_{Y}\right) Z=\left[\left(i_{X} \bullet i_{Y}\right)(Z)\right](\overleftarrow{\nabla} \bullet \overleftarrow{\nabla})
$$

Recall that in $\nabla_{X} Y$ first $\overleftarrow{\nabla}$ is carried out and afterwards $X$ acts as a contraction denoted by $i_{X}$. In addition, we have to respect the order of $\nabla_{X}$ and $\nabla_{Y}$. Indicating the order by a subscript, we have altogether

$$
\left(\nabla_{X} \bullet \nabla_{Y}\right) Z=\left[\left(i_{X(4)} \bullet i_{Y(2)}\right)(Z)\right]\left(\overleftarrow{\nabla}_{(1)} \bullet \overleftarrow{\nabla}_{(3)}\right)
$$

We apply the first covariant derivative by bringing $Z$ and $\overleftarrow{\nabla}_{(1)}$ together. The scalar product between $Z$ and the first matrix index of $\Gamma$ is carried out directly $Z \star \Gamma=Z^{M} \star \Gamma_{M}$ followed by bringing $i_{Y}$ together with $Z$. Thus, the computation proceeds as

$$
\begin{aligned}
& {\left[\left(i_{X(4)} \bullet i_{Y(2)}\right)(Z)\right]\left(\overleftarrow{\nabla}_{(1)} \bullet \overleftarrow{\nabla}_{(3)}\right)} \\
& =\left(i_{X(4)} \bullet i_{Y(2)}\right)^{\phi}\left[Z^{\phi}\left(\overleftarrow{\nabla}_{(1)} \bullet \overleftarrow{\nabla}_{(3)}\right)^{\phi}\right] \\
& =\left(i_{X(4)} \bullet i_{Y(2)}\right)^{\phi}\left[\partial Z^{\phi} \overleftarrow{\nabla}_{(3)}^{\phi}\right]+\left(i_{X(4)} \bullet i_{Y(2)}\right)^{\phi}\left[Z^{M \phi} \star\left(\Gamma_{M} \overleftarrow{\nabla}_{(3)}\right)^{\phi}\right] \\
& =\left[i_{X(4)}^{\phi}\left(Y^{\phi} \star \partial Z^{\phi}\right)\right] \overleftarrow{\nabla}_{(3)}+\left(\left[i_{X(4)}^{\phi}\left(Y^{\phi} \star Z^{M \phi}\right)\right]^{\bar{\phi}} \star \Gamma_{M}^{\bar{\phi}}\right) \overleftarrow{\nabla}_{(3)}^{\bar{\phi}} .
\end{aligned}
$$


Next, the second covariant derivative $\overleftarrow{\nabla}_{(3)}$ and afterwards $i_{X}$ are applied

$$
\begin{aligned}
= & i_{X(4)}^{\phi \phi^{\prime}}\left[\left(Y^{\phi} \star \partial Z^{\phi}\right) \phi^{\prime} \overleftarrow{\nabla}_{(3)}^{\phi^{\prime}}\right]+i_{X(4)}^{\phi \phi^{\prime}}\left[\left(Y^{\phi} \star Z^{K \phi}\right) \phi^{\phi^{\prime}} \star\left(\Gamma_{K} \overleftarrow{\nabla}_{(3)}\right) \phi^{\phi^{\prime}}\right] \\
= & X^{\phi} \star \partial\left(Y^{\phi} \star \partial Z^{\phi}\right)+\left[X^{\phi} \star\left(Y^{\phi} \star \partial Z^{K \phi}\right)\right] \star \Gamma_{K} \\
& +X^{\phi \phi^{\prime}} \star \partial\left[\left(Y^{\phi} \star Z^{K \phi}\right) \phi^{\phi^{\prime}} \star \Gamma_{K}^{\phi^{\prime}}\right]+\left[(X \star Y) \star Z^{K}\right] \star\left(\Gamma_{K}^{P} \star \Gamma_{P}\right) .
\end{aligned}
$$

In this formula we placed the brackets and the derivative $\partial$ in such a way that they reflect, which objects have to be contracted with each other. For instance in the first term of (B.5), the derivative is contracted with $X$. After applying the Leibniz rule for $\partial\left(Y^{\phi} \star \partial Z^{\phi}\right)$, this contraction must be kept in mind.

When computing the other terms in (B.1), one realizes that the first term in (B.5) is canceled partly by $\left(\nabla_{\overline{\mathcal{R}}(Y)} \bullet \nabla_{\overline{\mathcal{R}}(X)}\right) Z$ and partly by $\nabla_{[X, Y]_{\star}} Z$. The other term from $\nabla_{[X, Y]_{\star}} Z$ cancels the $X \star \partial Y \star Z \star \Gamma$ part in the third term of (B.5). The remaining two terms which have to cancel in (B.5) arise from the second and third term and are both of the form $X \star Y \star \partial Z \star \Gamma$. In one term $\partial$ is contracted with $Y$ and in the other $\partial$ is contracted with $X$. These terms cancel crosswise against similar terms appearing in $\left(\nabla_{\overline{\mathcal{R}}(Y)} \bullet \nabla_{\overline{\mathcal{R}}(X)}\right) Z$.

After all these cancellations, the Riemann-tensor (B.1) simplifies to

$$
\begin{aligned}
-R(X, Y, Z)= & \left((X \star Y) \star Z^{M}\right) \star \partial \Gamma_{M}+\left((X \star Y) \star Z^{M}\right) \star\left(\Gamma_{M}{ }^{P} \star \Gamma_{P}\right) \\
& -X \leftrightarrow^{\overline{\mathcal{R}}} Y .
\end{aligned}
$$

Recalling the discussion after (B.5), in the first term of (B.6), $X$ is contracted with $\partial$. This is in contrast to the rule that always a vector is contracted with the nearest neighboring form. ${ }^{11}$ To bring this into the usual notation, we switch the first term with its $\mathcal{R}$-permuted term and find with (4.21) and (4.22)

$$
\begin{aligned}
-R(X, Y, Z)= & -\left((X \star Y) \star Z^{M}\right) \star \partial \Gamma_{M}+\left((X \star Y) \star Z^{M}\right) \star\left(\Gamma_{M}^{P} \star \Gamma_{P}\right) \\
& -X \leftrightarrow^{\overline{\mathcal{R}}} Y .
\end{aligned}
$$

Now the notation matches the one in (5.22), where the vector $Y$ is contracted with the form $\partial$ (see also (5.26) and (5.27)). By utilizing (4.25) to transfer the antisymmetrization on the vector side towards the form side, we indeed find

$$
-R(X, Y, Z)=\left((X \star Y) \star Z^{K}\right) \star\left(-d \Gamma_{K}+\Gamma_{K}^{P} \wedge_{\star} \Gamma_{P}\right)
$$

This matches the definition of the star-Riemann curvature as the exterior covariant derivative of the connection $\Gamma$ in $(5.22)$.

Open Access. This article is distributed under the terms of the Creative Commons Attribution License (CC-BY 4.0), which permits any use, distribution and reproduction in any medium, provided the original author(s) and source are credited.

\footnotetext{
${ }^{11}$ Notice that the contraction in the second term $\sim \Gamma \star \Gamma$ comes out correctly according to this rule.
} 


\section{References}

[1] R. Blumenhagen and E. Plauschinn, Nonassociative Gravity in String Theory?, J. Phys. A 44 (2011) 015401 [arXiv: 1010.1263] [INSPIRE].

[2] D. Lüst, T-duality and closed string non-commutative (doubled) geometry, JHEP 12 (2010) 084 [arXiv: 1010.1361] [INSPIRE].

[3] P. Bouwknegt, K. Hannabuss and V. Mathai, Nonassociative tori and applications to T-duality, Commun. Math. Phys. 264 (2006) 41 [hep-th/0412092] [INSPIRE].

[4] E. Plauschinn, Non-geometric fluxes and non-associative geometry, PoS(CORFU2011) 061 [arXiv: 1203.6203] [INSPIRE].

[5] R. Blumenhagen, A Course on Noncommutative Geometry in String Theory, Fortsch. Phys. 62 (2014) 709 [arXiv: 1403.4805] [InSPIRE].

[6] R. Blumenhagen, A. Deser, D. Lüst, E. Plauschinn and F. Rennecke, Non-geometric Fluxes, Asymmetric Strings and Nonassociative Geometry, J. Phys. A 44 (2011) 385401 [arXiv: 1106.0316] [INSPIRE].

[7] C. Condeescu, I. Florakis and D. Lüst, Asymmetric Orbifolds, Non-Geometric Fluxes and Non-Commutativity in Closed String Theory, JHEP 04 (2012) 121 [arXiv:1202.6366] [INSPIRE].

[8] A. Chatzistavrakidis and L. Jonke, Matrix theory origins of non-geometric fluxes, JHEP 02 (2013) 040 [arXiv: 1207.6412] [INSPIRE].

[9] D. Andriot, M. Larfors, D. Lüst and P. Patalong, (Non-)commutative closed string on T-dual toroidal backgrounds, JHEP 06 (2013) 021 [arXiv: 1211.6437] [INSPIRE].

[10] C. Condeescu, I. Florakis, C. Kounnas and D. Lüst, Gauged supergravities and non-geometric Q/R-fluxes from asymmetric orbifold CFT's, JHEP 10 (2013) 057 [arXiv: 1307.0999] [INSPIRE].

[11] I. Bakas and D. Lüst, T-duality, Quotients and Currents for Non-Geometric Closed Strings, Fortsch. Phys. 63 (2015) 543 [arXiv:1505.04004] [INSPIRE].

[12] W. Siegel, Two vierbein formalism for string inspired axionic gravity, Phys. Rev. D 47 (1993) 5453 [hep-th/9302036] [INSPIRE].

[13] W. Siegel, Superspace duality in low-energy superstrings, Phys. Rev. D 48 (1993) 2826 [hep-th/9305073] [INSPIRE].

[14] C. Hull and B. Zwiebach, Double Field Theory, JHEP 09 (2009) 099 [arXiv:0904.4664] [INSPIRE].

[15] O. Hohm, C. Hull and B. Zwiebach, Background independent action for double field theory, JHEP 07 (2010) 016 [arXiv: 1003.5027] [INSPIRE].

[16] G. Aldazabal, D. Marques and C. Núñez, Double Field Theory: A Pedagogical Review, Class. Quant. Grav. 30 (2013) 163001 [arXiv: 1305.1907] [INSPIRE].

[17] D.S. Berman and D.C. Thompson, Duality Symmetric String and M-theory, Phys. Rept. 566 (2014) 1 [arXiv: 1306.2643] [INSPIRE].

[18] O. Hohm, D. Lüst and B. Zwiebach, The Spacetime of Double Field Theory: Review, Remarks and Outlook, Fortsch. Phys. 61 (2013) 926 [arXiv:1309.2977] [INSPIRE]. 
[19] R. Blumenhagen, M. Fuchs, F. Haßler, D. Lüst and R. Sun, Non-associative Deformations of Geometry in Double Field Theory, JHEP 04 (2014) 141 [arXiv: 1312.0719] [INSPIRE].

[20] R. Blumenhagen, F. Hassler and D. Lüst, Double Field Theory on Group Manifolds, JHEP 02 (2015) 001 [arXiv: 1410.6374] [INSPIRE].

[21] R. Blumenhagen, P.d. Bosque, F. Hassler and D. Lüst, Generalized Metric Formulation of Double Field Theory on Group Manifolds, JHEP 08 (2015) 056 [arXiv:1502.02428] [INSPIRE].

[22] A. Deser, Star products on graded manifolds and $\alpha^{\prime}$-corrections to Courant algebroids from string theory, J. Math. Phys. 56 (2015) 092302 [arXiv: 1412.5966] [INSPIRE].

[23] A. Deser, Star products on graded manifolds and $\alpha^{\prime}$-corrections to double field theory, in proceedings of the 34th Workshop on Geometric Methods in Physics (XXXIV WGMP), Bialowieza, Poland, June 28 - July 4 2015, arXiv:1511.03929 [INSPIRE].

[24] D. Mylonas, P. Schupp and R.J. Szabo, Membrane $\sigma$-models and Quantization of Non-Geometric Flux Backgrounds, JHEP 09 (2012) 012 [arXiv:1207.0926] [INSPIRE].

[25] I. Bakas and D. Lüst, 3-Cocycles, Non-Associative Star-Products and the Magnetic Paradigm of R-Flux String Vacua, JHEP 01 (2014) 171 [arXiv:1309.3172] [INSPIRE].

[26] D. Mylonas, P. Schupp and R.J. Szabo, Non-Geometric Fluxes, Quasi-Hopf Twist Deformations and Nonassociative Quantum Mechanics, J. Math. Phys. 55 (2014) 122301 [arXiv: 1312.1621] [INSPIRE].

[27] P. Aschieri, C. Blohmann, M. Dimitrijević, F. Meyer, P. Schupp and J. Wess, A gravity theory on noncommutative spaces, Class. Quant. Grav. 22 (2005) 3511 [hep-th/0504183] [INSPIRE].

[28] P. Aschieri, M. Dimitrijević, F. Meyer and J. Wess, Noncommutative geometry and gravity, Class. Quant. Grav. 23 (2006) 1883 [hep-th/0510059] [INSPIRE].

[29] L. Álvarez-Gaumé, F. Meyer and M.A. Vazquez-Mozo, Comments on noncommutative gravity, Nucl. Phys. B $\mathbf{7 5 3}$ (2006) 92 [hep-th/0605113] [INSPIRE].

[30] G.E. Barnes, A. Schenkel and R.J. Szabo, Nonassociative geometry in quasi-Hopf representation categories I: Bimodules and their internal homomorphisms, J. Geom. Phys. 89 (2014) 111 [arXiv:1409.6331] [INSPIRE].

[31] G.E. Barnes, A. Schenkel and R.J. Szabo, Nonassociative geometry in quasi-Hopf representation categories II: Connections and curvature, J. Geom. Phys. 106 (2016) 234 [arXiv: 1507.02792] [INSPIRE].

[32] G.E. Barnes, A. Schenkel and R.J. Szabo, Working with Nonassociative Geometry and Field Theory, [arXiv: 1601.07353] [INSPIRE].

[33] P. Aschieri and R.J. Szabo, Triproducts, nonassociative star products and geometry of R-flux string compactifications, J. Phys. Conf. Ser. 634 (2015) 012004 [arXiv:1504.03915] [INSPIRE].

[34] V.G. Kupriyanov, Alternative multiplications and non-associativity in physics, in proceedings of the 15th Hellenic School and Workshops on Elementary Particle Physics and Gravity (CORFU2015), Corfu, Greece, September 1-26, 2015, arXiv:1603.00218 [INSPIRE]. 